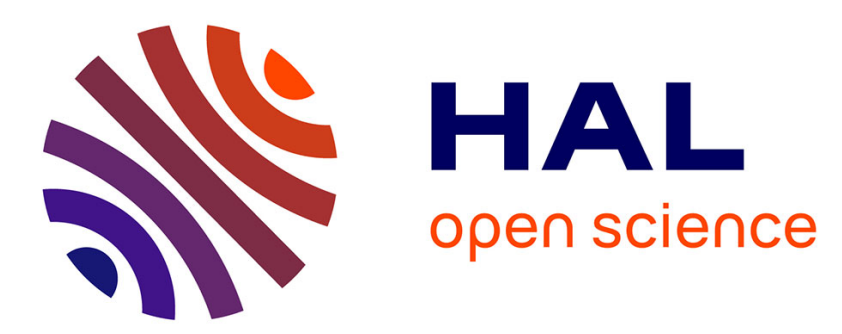

\title{
Efficacité économique et effets distributifs de long-terme des politiques de rénovation énergétique des logements
}

Louis-Gaëtan Giraudet, Cyril Bourgeois, Philippe Quirion

\section{To cite this version:}

Louis-Gaëtan Giraudet, Cyril Bourgeois, Philippe Quirion. Efficacité économique et effets distributifs de long-terme des politiques de rénovation énergétique des logements. Economie et Prévision, 2020. hal-03100351

\section{HAL Id: hal-03100351 \\ https://hal.science/hal-03100351}

Submitted on 12 Jan 2021

HAL is a multi-disciplinary open access archive for the deposit and dissemination of scientific research documents, whether they are published or not. The documents may come from teaching and research institutions in France or abroad, or from public or private research centers.
L'archive ouverte pluridisciplinaire HAL, est destinée au dépôt et à la diffusion de documents scientifiques de niveau recherche, publiés ou non, émanant des établissements d'enseignement et de recherche français ou étrangers, des laboratoires publics ou privés. 


\title{
Efficacité économique et effets distributifs de long-terme des politiques de rénovation énergétique des logements
}

\author{
Louis-Gaëtan Giraudet* (Ecole des Ponts ParisTech, CIRED) \\ Cyril Bourgeois (Ecole des Ponts ParisTech, CNRS, CIRED) \\ Philippe Quirion (CNRS, CIRED)
}

$2^{\text {ème }}$ version, 17 décembre 2019

\begin{abstract}
Nous évaluons, à l'aide du modèle technico-économique Res-IRF, l'effet des quatre principaux dispositifs de subvention à l'efficacité énergétique et de la taxe carbone sur la demande d'énergie pour le chauffage des logements en France. La taxe carbone s'avère être l'instrument le plus efficace, mais également le plus régressif. Les subventions induisent un effet levier sur l'investissement de 1 à 1,4 en 2015. Le ciblage des subventions sur les ménages à bas revenus, qui sont sur-représentés dans les logements les moins performants, accroît l'effet levier, permettant ainsi de concilier efficacité économique et équité sociale. L'atteinte des objectifs nationaux d'économie d'énergie nécessite de maintenir les subventions jusqu'en 2050 et de les étendre au parc locatif privé.
\end{abstract}

Mots clés: rénovation énergétique, taxe carbone, subventions à l'efficacité énergétique, précarité énergétique, évaluation intégrée, chauffage

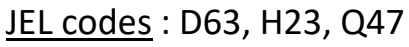

\footnotetext{
* Contact pour correspondance: giraudet@centre-cired.fr. Ces travaux ont bénéficié du soutien financier de l' Agence de l'environnement et de la maîtrise de l'énergie (Convention 1410 C 0001), du Commissariat général au développement durable (Convention 2101763372), de TOTAL S.A. et de l'Association Technique Energie Environnement (Convention DS N³136).
} 


\section{Long-term efficiency and distributional impacts of energy saving policies in the French residential sector}

Using the Res-IRF energy-economy model, we evaluate the impact of four key energy efficiency subsidy programmes and the carbon tax on energy demand for residential heating in France. The carbon tax proves to be the most effective, yet also most regressive, instrument. Subsidies have a leverage effect of 1 to 1.4 in 2015. Targeting subsidies towards low-income households, who tend to live in energy inefficient dwellings, increases leverage, thus reconciling economic efficiency and social equity. Meeting the long-term energy saving targets set by the French government requires maintaining subsidy programmes until 2050 and extended them to rented dwellings.

keywords: home energy retrofits, carbon tax, energy efficiency subsidies, fuel poverty, energy-economy modelling, space heating 


\section{Introduction}

Il est généralement admis que le secteur du bâtiment recèle un gisement d'économies d'énergie considérable et peu coûteux. Etayé par des études d'ingénieur - dont celle de McKinsey \& Co. (2009) est la plus emblématique -, cet argument a exercé une influence importante sur l'action publique, comme en témoigne le nombre et la diversité des politiques d'efficacité énergétique mises en place dans le secteur du logement au cours des dernières décennies. Initiée dans les années 1970 en réponse au premier choc pétrolier, la " chasse au gaspi » a d'abord fait la part belle aux mesures réglementaires, comme le passage à l'heure d'été en 1976 et les réglementations thermiques qui se sont succédées à partir de 1974 (Martin et al., 1998 ; Leray et de la Roncière, 2002). A partir des années 2000, la préoccupation croissante pour le changement climatique s'est traduite par une multiplication des programmes de subventions à l'efficacité énergétique dans le logement - crédit d'impôt pour le développement durable (devenu depuis le crédit d'impôt pour la transition énergétique, CITE) et taxe sur la valeur ajoutée réduite (TVAr) en 2005, certificats d'économies d'énergie (CEE) en 2006 et éco-prêt à taux zéro (EPTZ) en 2009. A ces instruments s'ajoute depuis 2014 la taxe carbone (TC), dont la progression a connu un coup d'arrêt en 2019 à la suite du mouvement des Gilets jaunes.

Aujourd'hui, ces politiques publiques doivent concourir à l'atteinte des objectifs d'amélioration de l'efficacité énergétique des logements définis dans le cadre de la Stratégie Nationale Bas-Carbone (SNBC), qui décline au niveau sectoriel les engagements de réduction des émissions de gaz à effet de serre pris par la France dans le cadre de l'Accord de Paris. La consommation d'énergie dans le logement est soumise aux objectifs suivants (IGF et CGEDD, 2017) :

- Objectif 1 : réduire la consommation d'énergie de $20 \%$ d'ici 2030 et de $50 \%$ d'ici 2050 par rapport aux niveaux de 2012.

- Objectif 2 : rénover 500000 logements chaque année, dont 120000 logements sociaux.

- Objectif 3 : éliminer les logements les moins efficaces - étiquettes $G$ et $F$ du diagnostic de performance énergétique (DPE) - d'ici 2025.

- Objectif 4 : améliorer la performance du parc de logements au niveau « bâtiment basse consommation ", soit un niveau minimum équivalent à l'étiquette B du DPE d'ici 2050.

- Objectif 5 : réduire la précarité énergétique de $15 \%$ d'ici 2020.

Face à un tel foisonnement de politiques publiques d'une part, d'objectifs à atteindre d'autre part, les questions suivantes s'imposent : Ces objectifs sont-ils compatibles entre eux ? Les politiques en place permettent-elles de les atteindre ? Si oui, à quel coût, et pour quels effets distributifs ?

Répondre à ces questions suppose de prendre en compte la complexité et l'hétérogénéité des déterminants de la consommation d'énergie dans le domaine du logement. En effet, l'optimisme des études d'ingénieur se heurte à un faisceau d'évidence révélant un écart systématique entre les économies d'énergie réalisables et réalisées - phénomène connu sous le terme d’Energy Efficiency Gap (Jaffe et Stavins, 1994 ; Allcott and Greenstone, 2012). Si les causes de ce phénomène sont multiples, les plus récurrentes incluent : l'effet rebond, ajustement comportemental transférant une partie des gains d'efficacité énergétique en amélioration du confort ; le dilemme propriétaire-locataire, qui implique une réticence des propriétaires à investir dans des technologies dont les bénéfices reviennent aux locataires ; 
les règles de copropriété qui ralentissent la décision collective dans l'habitat collectif ; l'accès hétérogène des ménages au crédit en fonction de leur revenu ; les coûts cachés de la rénovation énergétique, comme l'immobilisation du logement pendant les travaux de rénovation (Sorrell, 2004 ; Gillingham et al., 2009 ; Gerarden et al., 2017).

Dans cet article, nous évaluons la contribution des principales politiques de rénovation énergétique à I'atteinte des objectifs de la SNBC en utilisant Res-IRF, un modèle technico-économique qui vise précisément à décrire les principales barrières comportementales à l'amélioration énergétique des logements. Développé au CIRED depuis 2009 (Giraudet et al., 2012), le modèle Res-IRF a pour vocation d'améliorer le réalisme comportemental qui fait généralement défaut aux modèles intégrés de demande d'énergie (Mundaca et al., 2010, McCollum et al., 2017). Il se caractérise par une élasticité-prix de la demande d'énergie de -0,2 à court terme, -0,4 à long terme, et par un effet rebond de l'ordre de $20 \%$, soit des ordres de grandeur cohérents avec les estimations fréquemment rencontrées dans la littérature (Sorrell et al., 2009 ; Labandeira et al., 2017). La robustesse du modèle a été établie par une analyse de sensibilité dite globale (Branger et al., 2015) et par des rétro-simulations révélant une erreur de prédiction de moins de 1.5\% sur la période 1984-2012 (Glotin et al., 2019). Utilisé dans le cadre d'une évaluation des politiques du Grenelle de l'environnement (Giraudet et al., 2011), Res-IRF a permis d'établir que les politiques en place à l'époque étaient insuffisantes pour atteindre l'objectif ambitieux d'une réduction de la consommation des bâtiments existants de 38\% entre 2008 et 2020 - constat alors rejoint avec un outil similaire par Charlier et Risch (2012). Outre la mise à jour des objectifs et politiques à évaluer, l'exercice présenté ici se distingue de l'exercice de 2011 par deux approfondissements. D'une part, le modèle a été re-paramétré avec les données de l'enquête Phébus ${ }^{1}$, étude d'envergure permettant notamment de segmenter les ménages par niveau de revenu, et ainsi d'évaluer l'effet des politiques sur la précarité énergétique, mesurée ici par le nombre de ménages consacrant plus de $10 \%$ de leur revenu à l'énergie pour le chauffage. D'autre part, l'évaluation, qui se limitait précédemment à mesurer l'efficacité des politiques en termes de kilowattheures économisés, prend désormais en compte le coût des politiques, permettant ainsi d'en évaluer efficience, mesurée par les indicateurs de coûtefficacité (euro d'incitation publique par kilowattheure économisé) et d'effet levier (euro d'investissement supplémentaire par euro d'incitation publique). Ces nouveaux critères - efficience et précarité énergétique - permettent une meilleure appréciation des mérites respectifs des différents instruments.

Nous nous concentrons sur les politiques existantes à la couverture la plus large - CITE, TVAr, CEE, EPTZ et TC. Nous intégrons de plus la réglementation thermique de 2012 dans notre scénario de référence et simulons la réglementation plus ambitieuse prévue pour 2020. Nous ne tenons en revanche pas compte de certaines politiques importantes mais à l'éligibilité plus restreinte - comme les subventions de I'Agence nationale de l'habitat (Anah) ciblant les ménages à faible revenu - ou dont la modélisation est moins évidente - comme les campagnes d'information. L'évaluation s'opère dans un cadre d'équilibre partiel et porte spécifiquement sur la consommation d'énergie pour le chauffage des logements. Les

\footnotetext{
1 http://www.statistiques.developpement-durable.gouv.fr/sources-methodes/enquete-nomenclature/1541/0/enqueteperformance-lhabitat-equipements-besoins-usages.html
} 
bâtiments résidentiels représentant $26 \%$ de la demande finale d'énergie, dont $67 \%$ pour le chauffage, ce périmètre couvre $18 \%$ de la demande d'énergie finale en France.

Nous concluons que l'atteinte des différents objectifs nécessite de fixer les politiques à leur niveau le plus ambitieux et les maintenir jusqu'en 2050. Les programmes de subventions doivent être particulier être étendus au parc locatif privé. Un ciblage des aides inversement lié au revenu des ménages améliore l'efficience des aides tout en réduisant la précarité énergétique. L'équilibre budgétaire entre les recettes fiscales de la taxe carbone et le coût des subventions publiques est excédentaire à partir de 2025. La taxe carbone s'avère être l'instrument le plus efficace en termes d'économies d'énergie, mais aussi le plus régressif en termes d'incidence sur les différentes classes de revenu. Les subventions permettent de réaliser des économies d'énergie à un coût de 0,05 à 0,08 euro par kilowattheure cumulé actualisé, avec un effet levier sur les dépenses d'investissement de 0,9 à 1,4, soit un ordre de grandeur cohérent avec celui d'autres subventions publiques évaluées en France - par exemple le prêt à taux zéro (Gobillon et le Blanc, 2005). Le crédit d'impôt est la subvention la plus efficace, mais aussi la moins efficiente. Le modèle reproduit fidèlement les observations récentes de coût des politiques, à l'exception de l'éco-prêt à taux zéro, dont il surestime le coût d'un ordre de grandeur. Les interactions entre les politiques sont synergiques mais modestes, induisant un surcroît d'efficacité de l'ordre de $10 \%$.

Au-delà du cas spécifique de la France, l'exercice apporte un éclairage sur des questions économiques plus générales. D'abord, les synergies mises en évidence entre efficience économique et réduction de la précarité énergétique sont déterminées par la corrélation qui s'observe dans les données entre le revenu des ménages et l'efficacité des logements qu'ils occupent - une tendance a priori assez générale dans les États membres de l'UE. Ensuite, l'écart entre les effectifs d'EPTZ simulés et observés suggère que des barrières non prises en compte réduisent considérablement l'efficacité de ce dispositif. Dans la mesure où l'instrument possède des analogues dans d'autres pays, notamment en Allemagne, cette réflexion nous invite à envisager des travaux de microéconomie appliquée visant à identifier ces barrières. De plus, l'exercice montre que, dans le cadre d'investissements présentant une marge intensive - ici l'ampleur d'une rénovation énergétique - l'effet levier peut être supérieur à 1 malgré une faible additionnalité du nombre de participants - confirmée par ailleurs par d'autres études (Nauleau, 2014 ; Charlier et al., 2018). Ce résultat rappelle que la faible additionnalité des aides (ou participation infra-marginale en termes plus techniques) ne doit pas être systématiquement stigmatisée comme un " effet d'aubaine ". Enfin, en termes de modélisation, l'exercice propose une méthode originale pour quantifier l'effet propre d'un instrument au sein d'une panoplie de politiques, en explorant l'ensemble des combinaisons contrefactuelles possibles. La suite de l'article est organisée de la façon suivante. La section 2 donne un aperçu du modèle Res-IRF, en mettant en évidence les derniers développements. La section 3 propose une méthode d'évaluation et détaille le paramétrage des politiques. La section 4 présente les résultats. La section 5 discute les implications de politique publique et conclut. 


\section{Le modèle Res-IRF 3.0 en bref}

Le modèle Res-IRF simule la demande d'énergie pour le chauffage dans le secteur résidentiel en France. Il combine une description détaillée de la performance énergétique des logements et des caractéristiques socio-économiques des ménages qui les occupent. La dernière version du modèle (3.0) intègre une segmentation des ménages par niveau de revenu.

\subsection{Structure et données}

Le modèle est décrit en détail dans Giraudet et al. (2012) et Branger et al. (2015). La section ci-dessous donne un aperçu des processus clés et détaille les développements qui n'ont pas été inclus dans les références déjà publiées.

Le parc de logements est paramétré à partir des données de l'enquête Phébus, qui croise les données socio-économiques des ménages avec l'efficacité énergétique de leur logement. Le périmètre du modèle couvre les résidences principales en France métropolitaine, soit 23,9 millions de logements. Ce parc est segmenté en 1080 catégories, réparties de la façon suivante :

- Neuf catégories de performance énergétique, correspondant aux étiquettes $A$ à $G$ du diagnostic de performance énergétique (DPE) pour les logements construits avant 2012, et aux labels Bâtiment Basse Consommation (BBC) et Bâtiment à Energie Positive (BEPOS) pour les logements construits après 2012. Ces catégories décrivent implicitement les caractéristiques techniques de l'enveloppe (isolation, vitrage) et du système de chauffage.

- Quatre combustibles utilisés comme source principale de chauffage : électricité, gaz naturel, fioul domestique et bois-énergie (soit $91 \%$ de la demande d'énergie pour le chauffage).

- Deux catégories de logements : maison individuelle (61\%) et logement collectif (39\%).

- Trois catégories de propriétaires : propriétaires-occupants (61\%), propriétaires bailleurs (24\%) et bailleurs sociaux (15\%).

- Cinq niveaux de revenus pour les propriétaires et les occupants, proches ${ }^{2}$ des quintiles de revenus de la population française donnés par l'Insee (Figure 1).

${ }^{2}$ En considérant les bornes de revenu définies par l'INSEE, la catégorie $\mathrm{C} 1$ couvre $17 \%$ des ménages dans le modèle, la catégorie $\mathrm{C} 2$ couvre $19 \%, \mathrm{C} 3$ couvre $23 \%$, C4 couvre $22 \%$ et C5 couvre $19 \%$. 


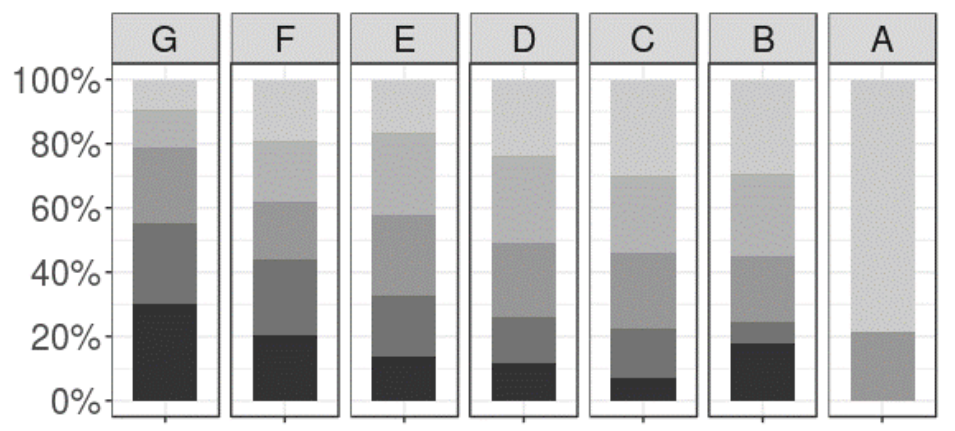

Catégorie de revenu $\square \mathrm{C} 1 \square \mathrm{C} 2 \square \mathrm{C} 3 \square \mathrm{C} 4 \square \mathrm{C} 5$

Figure 1: Distribution des catégories de revenu par niveau de performance énergétique

Le modèle est alimenté par trois variables d'entrée : la population, le revenu total et les prix des énergies. Ces variables déterminent l'amélioration de l'efficacité énergétique via la construction de logements neufs, la rénovation des logements existants et l'intensité avec laquelle les occupants utilisent leur infrastructure de chauffage.

\subsection{Construction neuve}

La construction de nouveaux logements est déterminée par :

- Une projection démographique exogène qui détermine les effectifs de logement. Cette projection est basée sur un scénario de l'Insee (2006) équivalente à un taux de croissance annuel moyen de $0,3 \%$.

- Une projection exogène du revenu des ménages, qui détermine la surface moyenne par logement. Combinée à la précédente, cette projection détermine les besoins de logement totaux en $\mathrm{m}^{2}$. On suppose que les revenus croissent à un taux annuel constant de $1,2 \%$, dans la poursuite des tendances récentes.

- Les nouvelles constructions sont déterminées chaque année comme le solde entre les besoins totaux et la part subsistante du parc existant (i.e., logements construits avant 2013, dont les effectifs s'érodent à un taux annuel constant de 0,35\% d'après Allaire et al. (2008)).

Cette spécification conduit à un stock cumulé de logements d'avant 2013 représentant $66 \%$ de la surface totale et $75 \%$ du nombre total de logements en 2050.

L'efficacité énergétique et l'énergie de chauffage principale de chaque nouveau logement sont déterminées conjointement par des fonctions de choix discret qui attribuent à chaque option une part de marché en fonction de son coût sur le cycle de vie, actualisé à $7 \%$ sur 35 ans. Le prix des énergie pris en compte dans ce calcul est celui de l'année considérée, traduisant des anticipations myopes bien 
documentées dans le secteur des transports (Anderson et al., 2013). Le tableau 1 détaille le coût de construction de chaque option (d'après CGDD, 2015)³.

Tableau 1: Coûts de construction, en $€ / \mathrm{m}^{2}$

\begin{tabular}{l|cc|cc}
\hline & \multicolumn{2}{|c|}{ Maison individuelle } & \multicolumn{2}{c}{ Logement collectif } \\
& BBC & BEPOS & BBC & 1308 \\
\hline Electricité & 979 & 1112 & 1199 & 1253 \\
Gaz naturel & 1032 & 1059 & 1242 & 1253 \\
Fioul domestique & 1032 & 1059 & 1242 & 1350 \\
Bois-énergie & 1094 & 1121 & 1323 & \\
\hline
\end{tabular}

\subsection{Rénovation des logements existants}

Les décisions de rénovation sont prises par les propriétaires - qu'ils soient occupants ou bailleurs du parc privé ou social. Pour un logement d'étiquette de départ $i$, les décisions opèrent sur deux marges :

- Marge intensive : sélection d'une étiquette finale $f$ parmi les étiquettes $\{i+1, \ldots, \mathrm{A}\}$. Ce processus est similaire à celui qui régit la construction neuve, avec des parts de marché de chaque option liée à son coût sur le cycle de vie par une fonction de choix discret. II présente toutefois quelques sophistications supplémentaires. D’abord, des taux d'actualisation négativement liés au revenu permettent de représenter les contraintes de crédit hétérogènes auxquelles font face les ménages (Tableau 2). Ensuite, les difficultés inhérentes à la délibération en copropriété sont représentées par des taux d'actualisation plus élevés dans les logements collectifs que dans les maisons individuelles (Tableau 2). De plus, la sous-capitalisation des économies d'énergie dans les loyers se traduit par un horizon d'investissement à court terme de trois ans dans le parc locatif privé, contre 30 ans dans les logements occupés par leur propriétaire et les logements sociaux. Enfin, des résidus sont calibrés en confrontant le modèle aux choix de rénovation observés; ces résidus sont interprétés comme des coûts intangibles, captant, par exemple, l'immobilisation du logement pendant les travaux de rénovation.

- Marge extensive : l'investisseur décide s'il rénove ou non son logement d'étiquette $i$ vers une étiquette $f>i$ plus élevée. Cette décision dépend de la valeur actuelle nette d'un projet de rénovation moyen, mesurée comme la différence de coût sur le cycle de vie entre le statu quo et les différentes options de rénovation pondérées par leur part de marché. La correspondance entre la valeur actuelle nette et les effectifs de rénovation suit une fonction logistique qui capte I'hétérogénéité des préférences et habitudes de chauffage. Il est calibré par rapport à un objectif de rénovation de $3 \%$ des logements existants en 2012.

Ces calculs reposent sur des données de coûts de rénovation intrinsèquement difficiles à obtenir. La représentation des rénovations énergétiques par des sauts de classe d'étiquette du DPE est en effet une abstraction de mesures explicites sur l'enveloppe et le système de chauffage. La matrice sur laquelle elle repose est construite à partir d'observations parcellaires, complétées par des données interpolées à partir des règles économiques standard de rendements décroissants - le coût incrémental de rénover à

\footnotetext{
${ }^{3}$ Les coûts de construction au niveau BBC sont plus élevés pour les logements chauffés à l'électricité que pour ceux chauffés avec d'autres combustibles, sous l'hypothèse que les premiers nécessitent une pompe à chaleur.
} 
un niveau $f+2$ par rapport à un niveau $f+1$ est supérieur au coût incrémental de rénover à un niveau $f+1$ par rapport à un niveau $f$ - et d'économies d'échelle - il est moins coûteux d'effectuer un même saut d'étiquette en une seule fois qu'en plusieurs fois. La matrice qui en résulte (Tableau 3) présente un coût moyen de 0,08 € par kilowattheure cumulé et actualisé sur la durée de vie.

Tableau 2: Taux d'actualisation, par type de logement et niveau de revenu des ménages

\begin{tabular}{c|c|c|c}
\hline Catégorie de revenu & Maison individuelle & Logement collectif & Logement social \\
\hline C1 & $15 \%$ & $37 \%$ & $4 \%$ \\
C2 & $10 \%$ & $25 \%$ & $4 \%$ \\
C3 & $7 \%$ & $15 \%$ & $4 \%$ \\
C4 & $5 \%$ & $7 \%$ & $4 \%$ \\
C5 & $4 \%$ & $5 \%$ & $4 \%$ \\
\hline Moyenne pondérée & $\mathbf{8 \%}$ & $\mathbf{1 7 \%}$ & $\mathbf{4 \%}$ \\
\hline
\end{tabular}

Tableau 3: Coûts de rénovation, en $€ / \mathrm{m}^{2}$

\begin{tabular}{|c|c|c|c|c|c|c|c|}
\hline & & \multicolumn{6}{|c|}{ Etiquette finale } \\
\hline & & $\mathbf{F}$ & $E$ & D & C & B & A \\
\hline \multirow{6}{*}{$\begin{array}{l}\text { Etiquette } \\
\text { initiale }\end{array}$} & $\mathbf{G}$ & 76 & 136 & 201 & 271 & 351 & 442 \\
\hline & $\mathbf{F}$ & & 63 & 130 & 204 & 287 & 382 \\
\hline & $\mathbf{E}$ & & & 70 & 146 & 232 & 331 \\
\hline & D & & & & 79 & 169 & 271 \\
\hline & C & & & & & 93 & 199 \\
\hline & B & & & & & & 110 \\
\hline
\end{tabular}

A titre d'illustration, en considérant une surface moyenne des logements de $91 \mathrm{~m}^{2}$, ces coûts s'échelonnent de $5733 €$ par logement (transition de $F$ vers $E$ ) à $40222 €$ (transition de $G$ à $A$ ), avec une moyenne à $112 € / \mathrm{m}^{2}$ soit $10192 €$. Les coûts de construction et de rénovation font l'objet diminuent de façon endogène grâce à des fonctions d'apprentissage paramétrées avec des taux d'apprentissage de $25 \%$ et $10 \%$, respectivement. 


\subsection{Comportement de chauffage}

En pratique, on observe un écart important entre les économies d'énergie prédites par les modèles de simulation thermique (tels que ceux utilisés dans le cadre du DPE) et réalisées (telles qu'elles se matérialisent par exemple sur la facture d'énergie). Ce phénomène dit de performance gap, mis en évidence dès l'évaluation des premiers programmes de rénovation énergétique (Hirst et Goeltz, 1985 ; Metcalf and Hassett, 1999) a connu un regain d'attention récemment (Fowlie et al., 2018). Trois explications sont généralement avancées : l'effet rebond direct, typiquement de l'ordre de $20 \%$ pour les consommations du chauffage (Sorrell et al., 2009) ; l'écart entre les hypothèses normatives des modèles de simulation et les conditions réelles d'utilisation, parfois désigné sous le terme de pre-bound effect (Sunikka-Blank et Galvin, 2012) ; et les asymétries d'information à l'origine d'une mauvaise qualité d'exécution des travaux (Giraudet et al., 2018).

L'écart de performance est pris en compte dans le modèle par une double comptabilité de la consommation d'énergie - réelle (telle qu'indiquée par les factures d'énergie) et conventionnelle (donnée par l'étiquette DPE). Le ratio entre les deux est interprété comme l'intensité avec laquelle les occupants - propriétaires-occupants et locataires - utilisent leur infrastructure de chauffage. Il évolue de façon endogène sous l'influence de trois variables : le prix de l'énergie, l'efficacité énergétique du logement (mesurée par son étiquette DPE) et le revenu du ménage occupant. Cette relation est déterminée par une fonction iso-élastique négative reliant l'intensité du chauffage à la part du revenu du ménage consacrée au chauffage, en reprenant une estimation de Cayla et Osso (2013) :

$$
\text { Intensité } d^{\prime} \text { utilisation }=-0,191 * \log (\text { Part budgétaire })+0,1105
$$

D’après cette relation, une amélioration de l'efficacité énergétique génère une augmentation de I'intensité de chauffage, processus qui peut s'interpréter comme un effet de rebond (cf. Aydin et al., 2017). Cette relation contribue également à l'élasticité-prix et à l'élasticité-revenu de la demande d'énergie du modèle. En l'absence de données empiriques systématiques sur le pre-bound effect et les problèmes de qualité, ces effets sont les seuls déterminants du performance gap pris en compte dans le modèle.

\section{Méthode d'évaluation}

\subsection{Scénario de référence}

Comme exposé précédemment, le modèle est alimenté par un scénario de croissance de la population de $0,3 \%$ par an et un scénario de croissance du revenu des ménages de 1,2 \% par an. Une troisième variable d'entrée détermine la dynamique du modèle : les prix des énergies. Ceux-ci sont basés sur un scénario défini par les autorités françaises (Ademe et DGEC), correspondant à un taux de croissance annuel moyen de 1,4 \% pour le gaz naturel, 2,2 \% pour le fioul domestique, 1,1 \% pour l'électricité et 1,2 $\%$ pour le bois-énergie (Figure 2), soit une évolution annuelle moyenne de l'indice de prix des énergies de $1,5 \%$. 


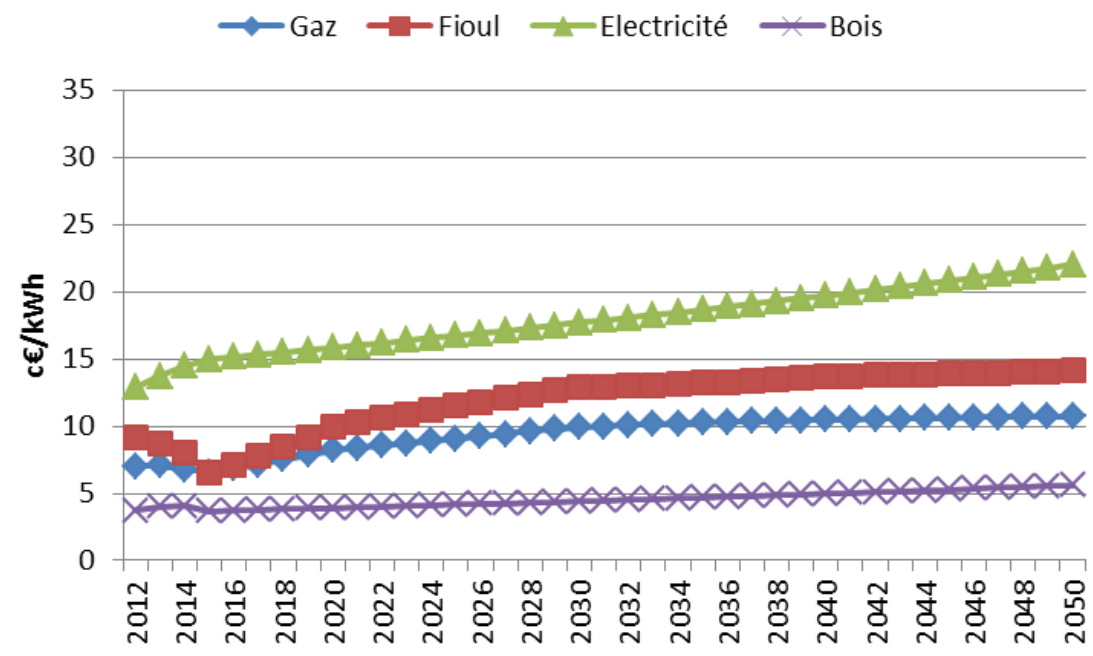

Figure 2: Scénario de prix des énergies (hors taxe carbone)

\subsection{Paramétrage des politiques}

L'étude se concentre sur les politiques de rénovation énergétique à la couverture la plus large. Pour chaque politique, deux cas sont considérés : la variante de référence et une variante plus ambitieuse, référencée « + ». Le paramétrage des politiques est résumé dans le Tableau 4.

Tableau 4: Principaux éléments de paramétrage des instruments

\begin{tabular}{l|c|c}
\hline & Variante TP & Variante TP+ \\
\hline CITE & Subvention 17\%, uniforme & Subvention 17\%, ciblée performance \\
\hline EPTZ & Subvention 8\%, ciblée performance & Subvention 22\%, ciblée performance \\
\hline CEE & $\begin{array}{c}\text { Subvention 4€/MWh cumac } \\
\text { (doublée pour ménages C1), puis 5€ en 2017 } \\
\text { croissant à 2\%/an, } \\
+ \text { taxe sur l'énergie }\end{array}$ & $\begin{array}{c}\text { Subvention 4€/MWh cumac } \\
\text { (doublée pour ménages C1), puis 15€ en 2018 croissant } \\
\text { à 2\%/an, } \\
+ \text { taxe sur l'énergie }\end{array}$ \\
\hline TC & Taxe profil LTECV, anticipation myope & Taxe profil LTECV, anticipation parfaite \\
\hline TVAr & \multicolumn{2}{|c}{ Taux de TVA de 5,5\% au lieu de 10\% } \\
\hline RT 2020 & \multicolumn{2}{|c}{ Niveau BEPOS seul autorisé à partir de 2020 } \\
\hline
\end{tabular}

\subsubsection{Taxe carbone (TC)}

La taxe carbone est modélisée à partir de 2014 avec la chronique définie par la loi de transition énergétique de 2017 (Tableau 5) ${ }^{4}$. Elle s'applique au fioul domestique et au gaz naturel, avec des contenus en carbone respectifs de 271 et $206 \mathrm{gCO} 2 / \mathrm{kWh}$. Ce dernier diminue à partir de 2020 au rythme de $1 \%$ par an pour tenir compte des objectifs d'incorporation de gaz d'origine renouvelable fixés par le gouvernement. La taxe carbone est soumise à une TVA de $20 \%$. Aucune hypothèse n'est faite quant à la réaffectation des recettes fiscales aux ménages.

4 Loi n²015-992 du 17 août 2015 relative à la transition énergétique pour la croissance verte. La taxe carbone ne s'applique pas à la consommation finale d'électricité, dont la production est couverte par le système communautaire d'échange des quotas d'émission. 
Tableau 4: Taxe carbone, en euros par tonne de $\mathrm{CO}_{2}$-equivalent (hors TVA)

\begin{tabular}{|c|c|c|c|c|c|c|c|c|}
\hline 2014 & 2015 & 2016 & 2017 & 2018 & 2019 & 2020 & $2020-30$ & $2030-50$ \\
\hline $7 €$ & $14,5 €$ & $22 €$ & $30,5 €$ & $39 €$ & $47,5 €$ & $56 €$ & $+6 \% /$ an, $100 €$ en 2030 & $+4 \% /$ an \\
\hline
\end{tabular}

Dans la variante de référence, la taxe carbone est, comme le prix de l'énergie, anticipée de façon myope. Dans la variante TC+, elle est en revanche parfaitement anticipée. Ces deux variantes visent à fournir une limite inférieure et supérieure au comportement des décideurs. II convient de rappeler qu'elles n'influencent que les décisions d'investissement, mais pas les comportements d'utilisation, qui sont fondés sur les signaux prix contemporains à la prise de décision.

A titre d'illustration, cette taxe représente (TVA incluse) en 2018 1,0c€/kWh pour le gaz naturel et $1,3 c € / \mathrm{kWh}$ pour le fioul domestique, soit respectivement $14 \%$ et $15 \%$ du prix (TTC) considéré cette année-là pour ces énergies.

\subsubsection{Eco-prêt à taux zéro (EPTZ)}

Le dispositif d'Eco-PTZ, mis en œuvre en 2009, permet aux ménages d'emprunter des fonds à taux d'intérêt nul auprès d'un établissement de crédit afin d'effectuer des travaux de rénovation énergétique de leur logement. L'organisme de prêt reçoit une compensation de l'Etat pour chaque prêt accordé. Le dispositif n'impose pas de restrictions sur les caractéristiques socio-économiques des emprunteurs, mais impose des restrictions sur la performance énergétique à réaliser. Ces exigences sont interprétées dans la modélisation comme un seuil de performance à atteindre fixé à l'étiquette $D\left(191 \mathrm{kWh} / \mathrm{m}^{2} / \mathrm{an}\right)$ pour la rénovation des étiquettes $G$ à $E$ et un seuil fixé à l'étiquette $B\left(76 \mathrm{kWh} / \mathrm{m}^{2} / \mathrm{an}\right)$ pour la rénovation des étiquettes $D$ et $C$.

L'instrument est modélisé comme une subvention égale aux intérêts d'un prêt d'un montant et d'une durée identiques contracté sur le marché du crédit à la consommation. La variante de référence vise à coller au plus près aux réalisations effectives du dispositif. Ainsi, elle intègre des plafonds d'emprunt correspondant aux moyennes par catégorie de revenu observées dans les statistiques produites par la Société de gestion des financements et de la garantie de l'accession sociale à la propriété (SGFGAS), l'autorité chargée du suivi du programme. De plus, la durée (cinq ans) et le taux d'intérêt (3\%) du prêt alternatif sont spécifiés à partir des résultats de l'enquête OPEN de l'Ademe (2016), qui précise les caractéristiques moyennes des prêts accompagnant les travaux de rénovation énergétique. Ces restrictions sont supprimées dans la variante EPTZ+, qui a pour but d'évaluer l'instrument comme s'il fonctionnait à son plein potentiel $l^{5}$. Pour un montant moyen d'emprunt de $10192 €$, ces spécifications représentent un montant d'intérêts de $796 €$ et $2191 €$, respectivement. Appliquées au coût moyen de rénovation dans le modèle, elles représentent une subvention ad valorem moyenne de $8 \%$ en référence et de $22 \%$ dans la variante EPTZ+. Les principales différences entre les deux scénarios sont récapitulées au Tableau 6.

\footnotetext{
${ }^{5}$ D'après le portail Webstat de la Banque de France, le taux d'intérêt des crédits à la consommation a chuté de façon régulière de 6,66\% au $1^{\mathrm{er}}$ janvier 2012 à 4,02\% au $1^{\text {er }}$ juin 2016 puis à 3,68\% au $1^{\text {er }}$ octobre 2019.
} 
Tableau 5: Paramétrage des variantes EPTZ

\begin{tabular}{|c|c|c|}
\hline & EPTZ & EPTZ+ \\
\hline Taux d'intérêt contrefactuel & 3\% (Ademe, 2016) & $4 \%$ \\
\hline Durée du prêt & 5 ans (Ademe, 2016) & 10 ans \\
\hline Plafond d'emprunt & $\begin{array}{c}21000 € \text { pour C5 } \\
\ldots \\
16800 € \text { pour C1 } \\
\text { (SFGAS) }\end{array}$ & $30000 €$ \\
\hline Ciblage de la performance & Oui & oui \\
\hline Taux de subvention équivalent & $9 \%$ & $23 \%$ \\
\hline
\end{tabular}

\subsubsection{Crédit d'impôt pour la transition énergétique (CITE)}

Mis en œuvre en 2005, le CITE accorde des réductions d'impôt sur le revenu pour les investissements dans la rénovation énergétique des logements (Nauleau, 2014). Dans son régime le plus récent, I'instrument prévoit un taux ad valorem fixe de $30 \%$. L'éligibilité s'applique au coût de fourniture des équipements et est étendue aux coûts d'installation pour les mesures d'isolation.

L'instrument est modélisé ici comme une subvention de $17 \%$ appliquée au coût total d'une mesure. Cette valeur constitue une approximation dans le temps et l'espace du taux de $30 \%$ sur la fourniture qui prévaut actuellement : dans le temps, elle constitue une moyenne des taux qui n'ont cessé de varier entre $15 \%$ et $50 \%$ au cours des années antérieures; dans l'espace, elle permet de tenir compte du fait que les coûts qui figurent dans le modèle sont des coûts complets, incluant fourniture et pose, et ce pour tous les équipements. La valeur de $17 \%$ est précisément calée sur le taux moyen de subvention déclaré par les ménages (Ademe, 2016) ; rapportée à l'investissement moyen de $10192 €$, elle s'élève à $1733 €$. Dans le scénario de référence, le même taux s'applique à toutes les mesures; dans la variante CITE+, le ciblage de I'EPTZ sur la haute performance s'applique.

\subsubsection{Certificats d'économies d'énergie (CEE)}

Les certificats d'économies d'énergie ont été mis en œuvre en France en 2006 (Giraudet et Finon, 2015). Le dispositif impose aux fournisseurs d'énergie des obligations d'économies d'énergie, qu'ils peuvent remplir en incitant leurs clients à prendre des mesures d'efficacité énergétique ou en achetant des économies d'énergie produites par d'autres fournisseurs obligés. Dans tous les cas, les économies d'énergie réalisées sont certifiées sur la base d'un montant forfaitaire en kWh dits " cumac »-cumulés (sur une durée de vie moyenne de 26 ans) et actualisés à 4\%. Les fournisseurs d'énergie peuvent, à des degrés divers selon le marché sur lequel ils opèrent, répercuter le coût de la contrainte dans leurs prix de vente d'énergie.

Le programme couvre tous les usages finaux de l'énergie non soumis au système communautaire d'échange des quotas d'émission (SCEQE) et prévoit une fongibilité totale des économies d'énergie entre secteurs. Un vendeur de carburant peut par exemple remplir son obligation en encourageant des mesures d'économie d'énergie dans le secteur du bâtiment, dont il répercutera le coût en augmentant le prix du carburant. A l'échelle d'un secteur, il n'existe donc pas de contrainte qui égalise le coût de réalisation d'une obligation et les compensations prélevées sur les ventes d'énergie. De même, le marché d'échange de certificats d'économies d'énergie est alimenté par réalisations provenant de différents secteurs. En théorie, le prix du marché devrait refléter le coût marginal du secteur où les 
économies d'énergie sont les plus dures à réaliser. En pratique, d'après les observateurs du marché, le prix est plutôt déterminé par la spéculation sur les objectifs futurs d'une part, et par la mise en réserve des économies d'énergie pour remplir ses obligations futures d'autre part. Les fluctuations de court terme du marché sont donc vraisemblablement déconnectées du coût marginal des mesures d'économie d'énergie.

Pour cette raison, modéliser de l'impact de l'instrument dans les bâtiments résidentiels nécessite d'importantes simplifications. L'instrument est modélisé ici comme un mécanisme hybride (Giraudet et Quirion, 2008), avec les composantes suivantes:

- Subvention à la rénovation énergétique, proportionnelle aux économies d'énergie générées par le saut d'étiquette considéré, actualisées à $4 \%$ sur 15 ans, considéré comme la durée de vie moyenne des opérations CEE. La proportionnalité de la subvention est donnée par le prix des certificats d'économie d'énergie (en $€ / k W h$ cumac), qui fait l'objet d'une scénarisation (cf. supra).

- Taxe sur l'énergie donnée par les coefficients d'obligation officiels (en kWh cumac à économiser par kWh vendu, augmentant par hypothèse de $1 \%$ par an après 2020) multipliés par le prix des certificats d'économies d'énergie, à quoi s'ajoute une TVA de $20 \%$.

Le prix des certificats blancs est fixé à $4 € / M W h$ cumac de 2012 à 2015. Il diverge en 2016 dans les deux variantes $-5 €$ en CEE et $15 €$ en CEE+. Pour les années suivantes, ces prix augmentent à un taux annuel de $2 \%$ et est plafonné à $20 € / \mathrm{MWh}$ cumac $^{6}$. A titre indicatif, le prix de $4 € / M W h$ cumulé sur 15 ans et actualisé à $4 \%$ représente une subvention ad valorem au barème décroissant (Tableau 7), représentant en moyenne $5 \%$ des coûts de rénovation exposés au Tableau 3, soit $510 €$ pour un montant d'investissement moyen de $10192 €$.

Tableau 6: Taux de subvention équivalent pour les CEE (pour un prix de 4€/MWh cumac, appliqué au coûts du Tableau 3)

\begin{tabular}{|c|c|c|c|c|c|c|}
\hline & $\mathrm{F}$ & $E$ & D & C & B & $A$ \\
\hline G & $11 \%$ & $10 \%$ & $8 \%$ & $7 \%$ & $6 \%$ & $5 \%$ \\
\hline $\mathrm{F}$ & & $8 \%$ & $6 \%$ & $5 \%$ & $4 \%$ & $3 \%$ \\
\hline$E$ & & & $5 \%$ & $4 \%$ & $3 \%$ & $2 \%$ \\
\hline$D$ & & & & $3 \%$ & $2 \%$ & $2 \%$ \\
\hline C & & & & & $2 \%$ & $1 \%$ \\
\hline B & & & & & & $1 \%$ \\
\hline
\end{tabular}

\subsubsection{TVA à taux réduit (TVAr)}

Les mesures de rénovation sont soumises à une TVA réduite de 5,5\%, au lieu du taux normal de $10 \%$ qui s'applique dans le secteur du bâtiment. Cette hypothèse est directement intégrée à la matrice de coûts.

\footnotetext{
${ }^{6}$ Le prix plafond correspond au montant de la pénalité libératoire du dispositif. Pendant longtemps, cette pénalité a été fixée à $20 € / M W h$ cumac. Elle a été abaissée en 2016 à 15€/MWh cumac. Néanmoins, en raison de la fiscalité qui s'applique sur la pénalité au cas où celle-ci doit être acquittée, le coût d'opportunité d'un manquement à l'objectif est supérieur à $15 € / M W h$ cumac. Pour prendre en compte ces différents éléments, on fixe le prix plafond à $20 € / M W h$ cumac.
} 


\subsubsection{Réglementation thermique (RT2020)}

Le scénario de référence du modèle intègre le niveau $B B C$ comme norme réglementaire depuis 2012 et fixe cette norme au niveau BEPOS à partir de 2020.

\subsection{Critères d'évaluation}

Quatre scénarios clés sont simulés :

- "Toutes politiques » (TP) : incorpore toutes les politiques dans leur variante de référence. C'est le scénario sur lequel est calibré le modèle.

- $\mathrm{TP}+$ : intègre toutes les politiques dans leur variante $«+»$.

- "Zéro politique » (ZP) : toutes les politiques sont supprimées.

- «Toutes politiques, sans dilemme propriétaire-locataire » (TP-DPL) : I'horizon d'investissement des propriétaires bailleurs est aligné sur celui des propriétaires occupants.

Les subventions sont toutes cumulables entre elles. Nous faisons en outre l'hypothèse forte qu'elles fonctionnent à plein régime, c'est-à-dire que les ménages ont une connaissance parfaite des programmes d'aide et que, par conséquent, toute mesure éligible va effectivement donner lieu à subvention.

\subsubsection{Choix d'une situation contrefactuelle}

Chaque scénario combinant plusieurs politiques, une multitude de situations contrefactuelles peuvent être envisagées pour estimer l'effet propre de chaque politique. Deux cas polaires délimitent l'effet d'un instrument donné. D’une part, le scénario de référence qui inclut toutes les politiques (TP) peut être comparé à un scénario qui inclut tous les instruments sauf un. Cette méthode, que nous désignons par l'abréviation "TP-1 », permet d'évaluer l'effet d'un instrument en interaction avec tous les autres instruments. D'autre part, un scénario dans lequel toutes les politiques ont été supprimées (ZP) peut être comparé à un scénario dans lequel une seule politique a été ajoutée. Cette méthode, que nous désignons par l'abréviation « ZP+1 », permet d'évaluer l'effet pur de l'instrument, indépendamment des autres instruments. A partir de ces deux cas polaires, une multitude de comparaisons peuvent être faites entre un scénario TP- $k$ et un scénario TP- $(k+1)$ (ou, de manière équivalente, entre $Z \mathrm{P}+k$ et ZP+k+1) pour quantifier l'interaction du ( $k+1)$-ième instrument avec $k$ autres. En considérant toutes les combinaisons possibles, 64 scénarios peuvent être exécutés (en incluant TP et ZP) et l'impact de chaque instrument peut être évalué de 32 manières différentes.

\subsubsection{Coût-efficacité}

L'indicateur de coût-efficacité est une première mesure de l'efficience d'un instrument. Il est calculé, pour une année donnée, comme le rapport entre le coût pertinent de l'instrument - dépense publique de subventions ou recette fiscale de taxe cette année-là - et les économies d'énergie générées, évaluées en comparant un scénario avec l'instrument (et éventuellement d'autres) à celui dans lequel l'instrument est retiré cette année-là (mais maintenu pour les années précédentes). Deux variantes de cet indicateur sont calculées pour tenir compte des différences entre économies d'énergie conventionnelles et réelles. La comparaison des deux permet d'estimer l'importance de l'effet rebond. Dans les deux cas, les 
économies d'énergie sont actualisées à $4 \%$ sur 26 ans, soit la durée de vie moyenne des investissements d'efficacité énergétique.

\subsubsection{Effet levier}

L'effet levier constitue une deuxième mesure d'efficience. Il est calculé comme le rapport entre le coût pertinent d'un instrument et les dépenses supplémentaires de rénovation qu'il induit, évaluées en comparant les scénarios avec et sans l'instrument en place cette année-là (mais dans les deux cas présents toutes les années précédentes). Ainsi défini, l'indicateur agrège les effets sur les marges intensives et extensives d'investissement.

Un effet levier élevé et un faible rapport coût-efficacité reflètent tous deux une efficience élevée. Ils recouvrent toutefois des phénomènes sensiblement différents, car les économies d'énergie (sur lesquelles est basé l'indicateur coût-efficacité) sont marginalement décroissantes tandis que les coûts marginaux (sur lesquels est basé l'effet levier) sont marginalement croissants.

\subsubsection{Précarité énergétique}

L'indicateur retenu ici pour évaluer la précarité énergétique est le taux d'effort énergétique (TEE), qui recense le nombre de ménages consacrant plus de $10 \%$ de leur revenu aux dépenses de chauffage. Ce critère recouvre 2,7 millions de ménages dans le modèle pour l'année 2012 , soit une estimation très proche de celle de l'Observatoire de la précarité énergétique (ONPE, 2016). Si d'autres indicateurs sont possibles (Charlier et Legendre, 2019), des simulations préliminaires non reportées ici indiquent qu'ils conduisent à taux de réduction de la précarité énergétique qualitativement similaires à ceux obtenus avec le TEE.

\section{Résultats}

\subsection{Efficacité environnementale}

La Figure 3 illustre la consommation d'énergie finale dans les différents scénarios. La consommation réelle d'énergie diminue de $18 \%$ en 2020 et de $45 \%$ en 2050 par rapport à 2012 avec toutes les politiques (TP). L'objectif 1 n'est donc pas atteint. Il l'est lorsque les politiques sont fixées à des niveaux plus ambitieux (TP+) ou étendues au parc locatif privé (TP-DPL). Rappelons toutefois que l'objectif 1 s'applique à la consommation totale d'énergie française. Appliqué spécifiquement au secteur du bâtiment résidentiel, qui connaît historiquement la plus forte diminution de consommation d'énergie (environ 1\%/an, d'après ADEME, 2015), cet objectif est par nature peu ambitieux. En comparant les scénarios TP et ZP, on constate que l'essentiel des économies d'énergie est dû à l'amélioration autonome de l'efficacité énergétique, stimulée notamment par la hausse des prix des énergies", le remplacement naturel des équipements défectueux et la RT2012. Les politiques contribuent pour environ un tiers aux économies d'énergie. Une observation plus détaillée révèle que la plupart des réductions portent sur les combustibles fossiles - gaz naturel et, dans une moindre mesure, fioul domestique. L'augmentation de

\footnotetext{
${ }^{7}$ Une comparaison (non reproduite ici) du scénario ZP à un scénario sans aucune politique et où les prix des énergies sont gelés à l'année de base permet de conclure que la hausse des prix des énergies de 1,5\% par an en moyenne contribue à la moitié des réductions autonomes de consommation d'énergie.
} 
I'intensité d'utilisation d'environ $20 \%$ au cours de la période dans les scénarios ZP et TP (Figure 4) peut être interprétée comme un effet rebond de $20 \%$.



Figure 3: Consommation d'énergie finale

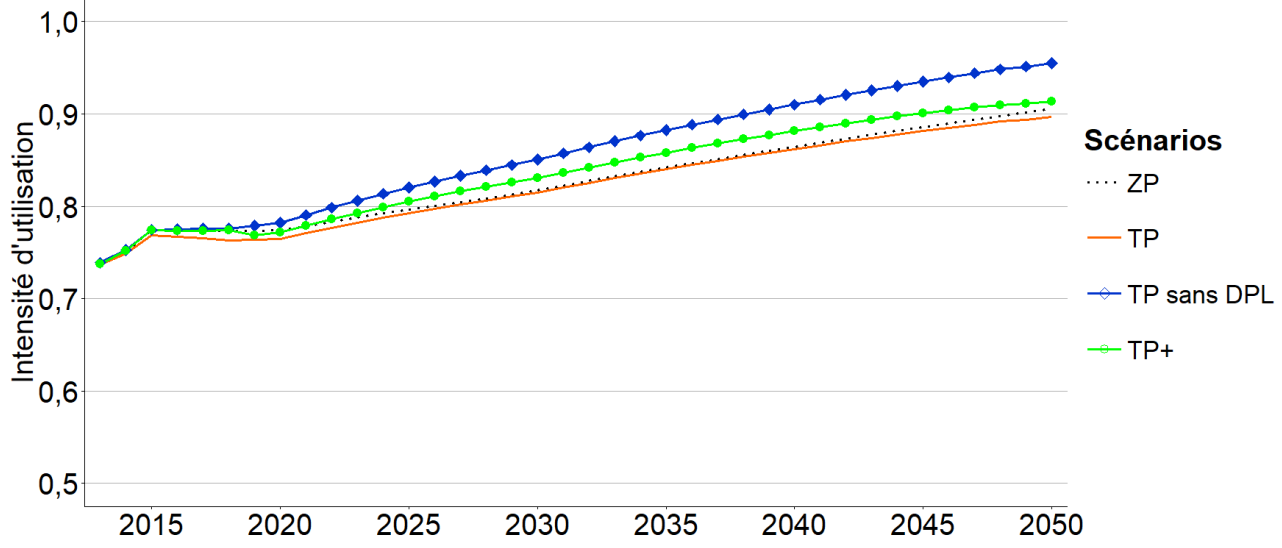

Figure 4: Intensité d'utilisation

La dynamique de rénovation est décrite à la Figure 5. Le nombre de rénovations s'élève à 687000 en 2012, dont 115000 sont attribuables aux politiques ${ }^{8}$. A première vue, l'objectif 2 est atteint, en l'absence même de toute politique. Deux nuances s'imposent néanmoins. D'abord, le nombre de rénovations simulées dans le logement social s'élève à 40000 , soit $6 \%$ du total des rénovations, alors que l'objectif 2 fixe cette part à $24 \%$. Ensuite, est comptabilisé ici comme rénovation énergétique tout saut d'au moins une étiquette du DPE. En pratique, la métrique qui devrait servir à évaluer l'objectif de 500000

${ }^{8}$ Un examen plus fin de cette dynamique (non détaillé ici) révèle que le nombre de sauts d'une seule étiquette s'élève à 355000 en 2015. Cette valeur est cohérente avec l'ordre de grandeur de 1,04 million sauts d'une étiquette recensés dans l'enquête TREMI de l'Ademe (2018) pour les seules maisons individuelles au cours de la période 2014-2016. 
rénovations énergétiques n'est définie nulle part, rendant l'évaluation de l'objectif 2 impraticable. Quoi qu'il en soit, le nombre de rénovations augmente dans le scénario TP+ et plus encore dans le scénario TP-DPL. Étant donné que ces deux scénarios génèrent des économies d'énergie comparables (cf. Figure 3), la différence observée sur le nombre de rénovations indique que le scénario TP agit principalement sur la marge intensive d'investissement alors que le scénario TP-DPL agit davantage sur la marge extensive.

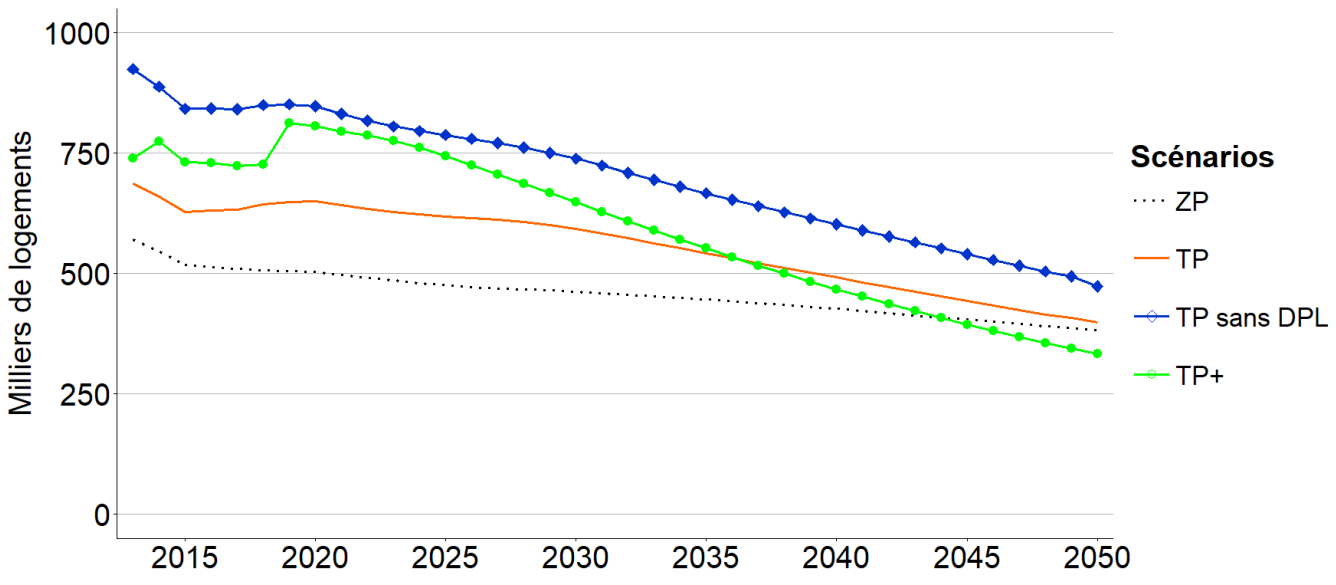

Figure 5: Dynamique de rénovation énergétique 
L'évolution de la performance énergétique des logements est décrite à la Figure 6 . Les étiquettes $G$ et $F$, généralement considérées comme des " passoires thermiques ", suivent une évolution très similaire dans les scénarios ZP, TP et TP+. Leurs effectifs diminuent de $75 \%$ entre 2012 et 2025 et plus lentement par la suite. L'objectif 3, qui prévoit l'élimination de ces étiquettes d'ici 2025, semble donc hors d'atteinte. Si la résolution du dilemme propriétaire-locataire (TP-DPL) induit de nets progrès par rapport à cet objectif, la quasi-disparition des étiquettes $F$ et $G$ ne semble pas envisageable avant 20409. A l'autre extrémité du spectre, les logements les plus performants - étiquettes B et A du stock pré-2012 et BBC et BEPOS du stock post-2012 - représente, selon le scénario considéré, entre 50 et $70 \%$ du parc total de 2050. Ces chiffres sont nettement en-deçà de l'objectif de 100 \% (objectif 4).

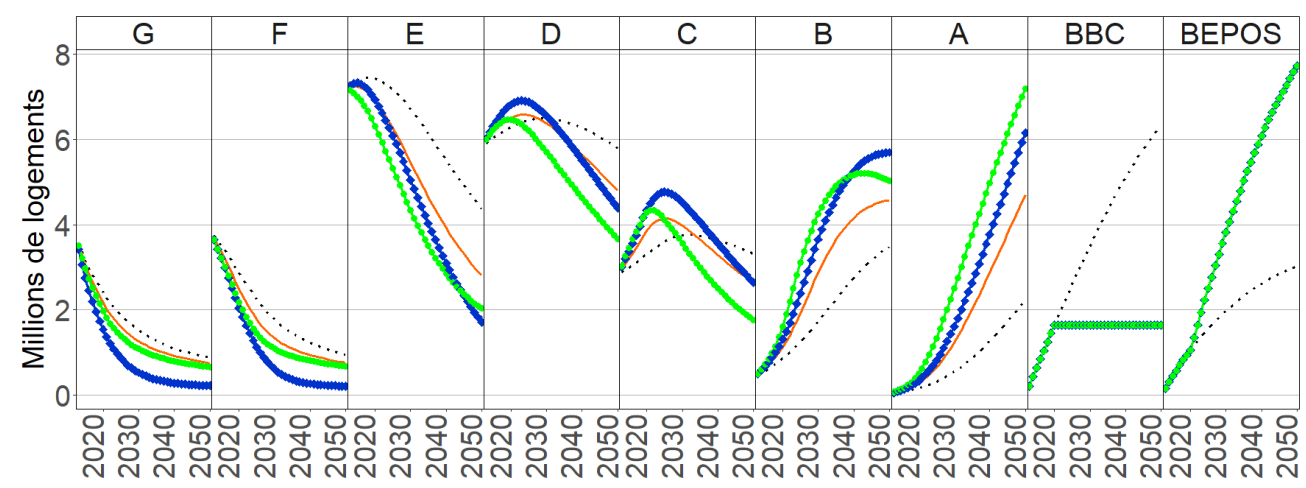

Scénarios $\cdots \mathrm{ZP}-\mathrm{TP} \multimap \mathrm{TP}$ sans $\mathrm{DPL}-\mathrm{TP}+$

Figure 6: Evolution de la performance énergétique du parc de logements

La Figure 7 illustre l'évolution de l'indice de TEE, qui recense les ménages consacrant plus de $10 \%$ de leur revenu au chauffage. Ainsi mesurée, la précarité énergétique diminue de deux tiers à l'horizon 2050 dans le scénario ZP. Le scénario TP freine ce processus, avec une réduction de seulement $7 \%$ à l'horizon 2020, valeur inférieure au seuil de $15 \%$ de l'objectif 5 . En revanche, le scénario TP+ et, dans une plus large mesure, TP-DPL, accélèrent le processus et permettent d'atteindre l'objectif. Dans la mesure où I'indicateur TEE prend en compte les dépenses d'énergie, la différence qu'il génère entre le scénario TP et les scénarios TP+ et TP et TP-DPL s'explique par des niveaux de subvention plus importants dans ces derniers $\operatorname{cas}^{10}$. La réduction plus lente observée dans le scénario TP peut donc être attribuée au poids relativement plus important de la taxe carbone dans le bouquet de politiques. Ce résultat confirme l'effet régressif d'une taxe carbone non recyclée aux ménages, bien documenté par ailleurs (Combet et al., 2010). L'effet régressif de la taxe carbone s'annule lorsque celle-ci s'accompagne de subventions fixées à des taux suffisamment ambitieux (TP+), ou étendues au parc locatif privé (TP-DPL), au point de laisser entrevoir une disparition de la précarité énergétique d'ici 2050. Outre la diminution de la part des

${ }^{9}$ Le modèle intègre, pour chaque étiquette DPE, une contrainte qui empêche les effectifs de chaque étiquette de passer sous le seuil de 5\% de leur niveau de 2012. Ce seuil vise à capter les contraintes architecturales ou urbaines.

${ }^{10}$ L'effet de la taxe carbone sur les dépenses énergétiques est très proche dans les scénarios TP et TP+, puisqu'il n'est pas directement affecté par l'hypothèse d'anticipation. 
dépenses consacrées au chauffage, les niveaux de confort thermique, mesurés par l'intensité d'utilisation, augmentent à un rythme similaire pour toutes les catégories de revenus, à partir toutefois de niveaux initiaux fortement corrélés au revenu (Figure 8). Ce résultat rappelle que la réduction de la précarité énergétique et la réduction des inégalités ne vont pas nécessairement de pair.

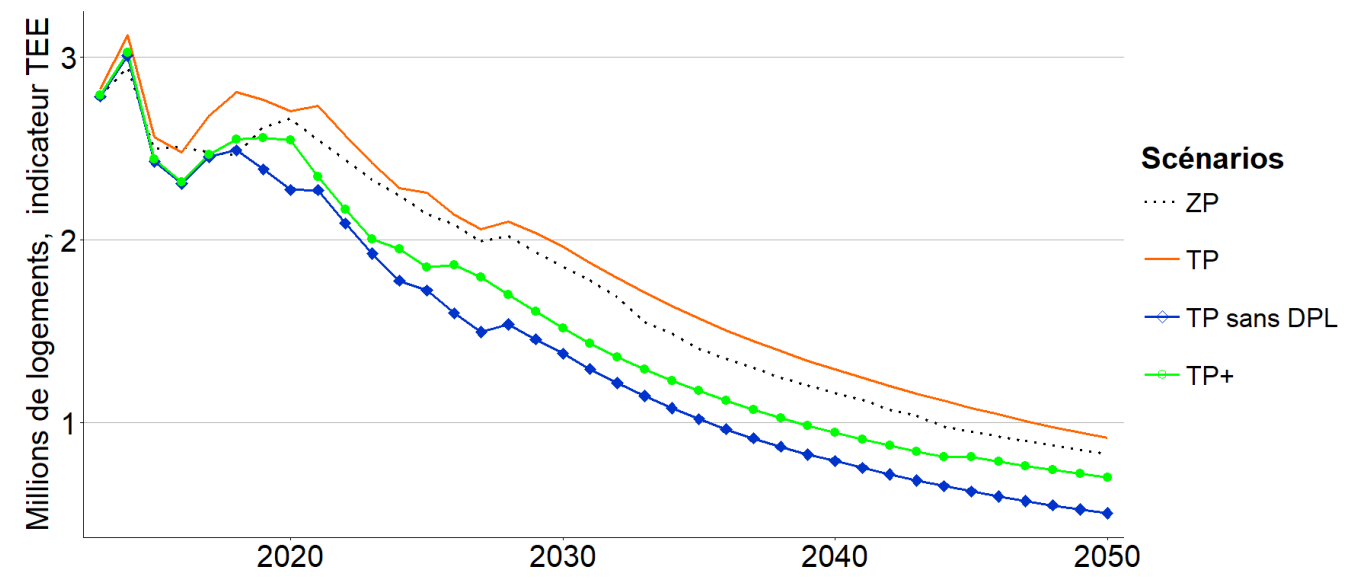

Figure 7: Evolution de la précarité énergétique

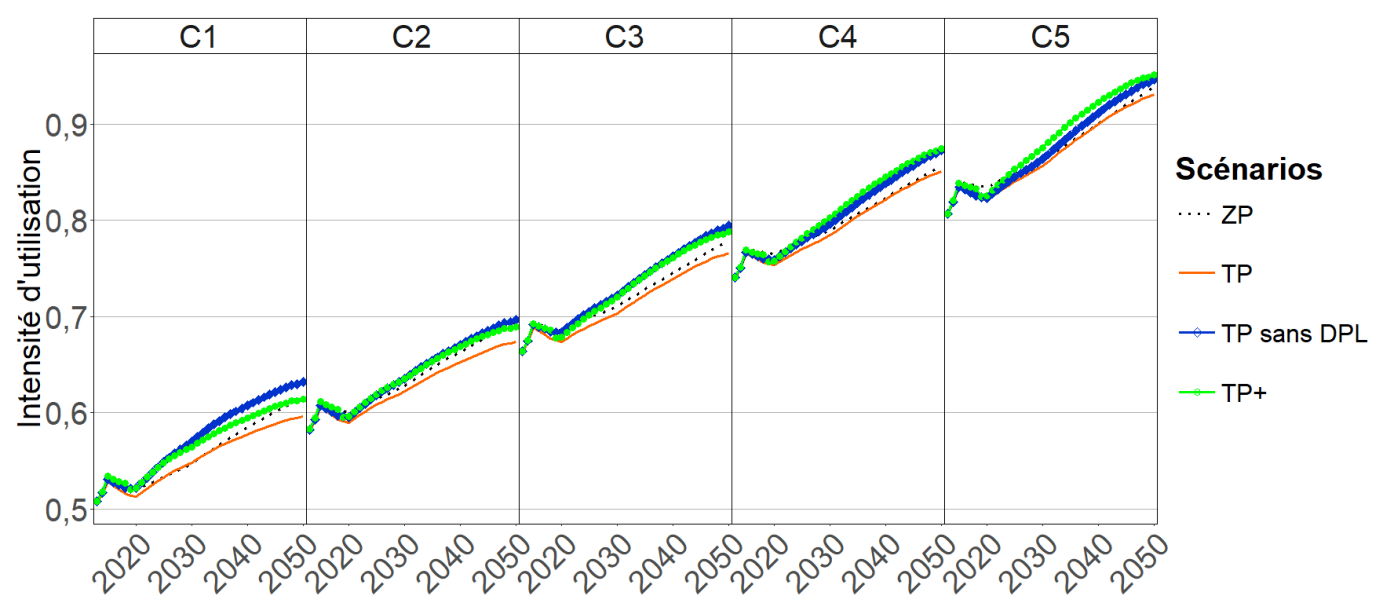

Figure 8: Intensité d'utilisation, par niveau de revenu

Dans l'ensemble, notre évaluation suggère qu'atteindre les différents objectifs nécessite de fixer les politiques à leur niveaux les plus ambitieux et les maintenir jusqu'en 2050. 


\subsection{Efficience comparée}

Après avoir évalué l'atteinte des objectifs en considérant différents bouquets de politiques publiques, on isole ici l'impact de chaque politique à partir de la méthode exposée au paragraphe 3.3.1. La Figure 9 compare les économies d'énergie réalisées par chaque instrument, calculées à partir des 32 combinaisons de politiques possibles impliquant ce même instrument. Les histogrammes indiquent les économies moyennes, les barres indiquent l'écart-type et les cas polaires TP-1 et ZP+1 sont identifiés par des marqueurs spécifiques. La taxe carbone s'avère être l'instrument le plus efficace. Cette performance s'explique par la combinaison de deux effets : une incitation à la rénovation associée à une incitation à réduire les dépenses énergétiques après rénovation. En comparaison, les subventions ne mobilisent que le premier levier. De plus, la taxe carbone augmente par construction au fil du temps, alors que les subventions (à l'exception des CEE, qui sont de toute façon fixées à un niveau faible) sont maintenues à un niveau constant. Le CITE est la subvention à l'impact le plus fort, en raison de son taux relativement élevé. Le fait que les économies d'énergie dans le scénario TP-1 soient systématiquement supérieures à celles du scénario ZP+1 suggère l'existence d'interactions sur-additives entre instruments. Dans l'ensemble, les interactions entre les politiques induisent une variation de $10 \%$ de l'efficacité d'un instrument. Ce niveau relativement modeste nous conduit à nous concentrer sur la méthode TP-1 - qui correspond a priori à la situation la plus proche de la réalité - dans les analyses ultérieures.

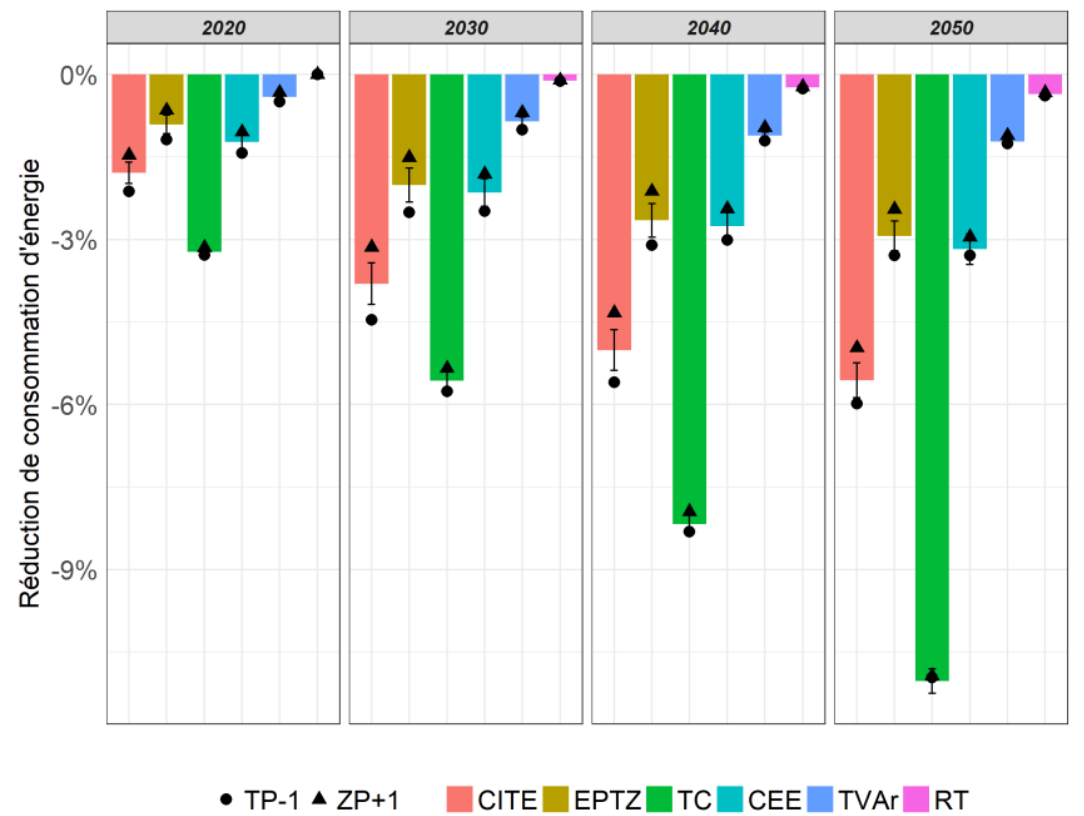

Figure 9: Efficacité des politiques en termes d'économies d'énergie

La Figure 10 compare le coût des subventions publiques tels que simulé par le modèle à ceux estimés par I'Inspection générale des finances (IGF et CGEDD, 2017). Les ordres de grandeur sont remarquablement cohérents entre les deux estimations pour la TVAr et, dans une moindre mesure, pour le CITE ; pour ce dernier, le moindre coût obtenu dans les simulations s'explique par le fait que la modélisation ignore certaines mesures éligibles au CITE, comme les petits équipements renouvelables. En revanche, les deux estimations de coût diffèrent considérablement pour l'EPTZ. Cet écart est confirmé par un écart d'un 
ordre de grandeur entre le nombre annuel d'EPTZ simulés - 400000 - et les EPTZ effectivement contractés - 40.000 en moyenne enregistrés entre 2013 et 2016 par l'autorité qui suit le dispositif (SGFGAS). Un tel écart met en évidence des barrières potentielles non prises en compte dans la modélisation : du côté de la demande, on pense à des barrières cognitives qui empêchent les emprunteurs d'exprimer les gains d'intérêts en subvention équivalente ; du côté de l'offre, on peut imaginer un comportement stratégique des établissements de crédit, qui privilégient leurs offres commerciales de crédit à la consommation au détriment des offres d'EPTZ. Ces questions constituent une perspective de recherche intéressante en microéconomie appliquée. Malgré cette limite, les projections de coûts suggèrent que, toutes politiques confondues - qu'elles soient financées par le secteur public (TC, EPTZ, CITE, TVAr) ou privé (CEE) - les recettes de taxe sur l'énergie dépasseront les dépenses de subventions à partir 2025 (Figure 11) ${ }^{11}$. Ce résultat ouvre la voie à une coordination plus étroite entre dépenses et recettes fiscales liées à la rénovation énergétique. Le coût net des polices s'élève à 3 milliards d'euros pour la première année 2012. Dans le même temps, l'investissement total dans la rénovation énergétique résidentielle s'élève à 7,5 milliards d'euros dans le scénario ZP, 10 milliards dans le scénario TP et 12,5 milliards dans les deux scénarios TP+ et TP-DPL.

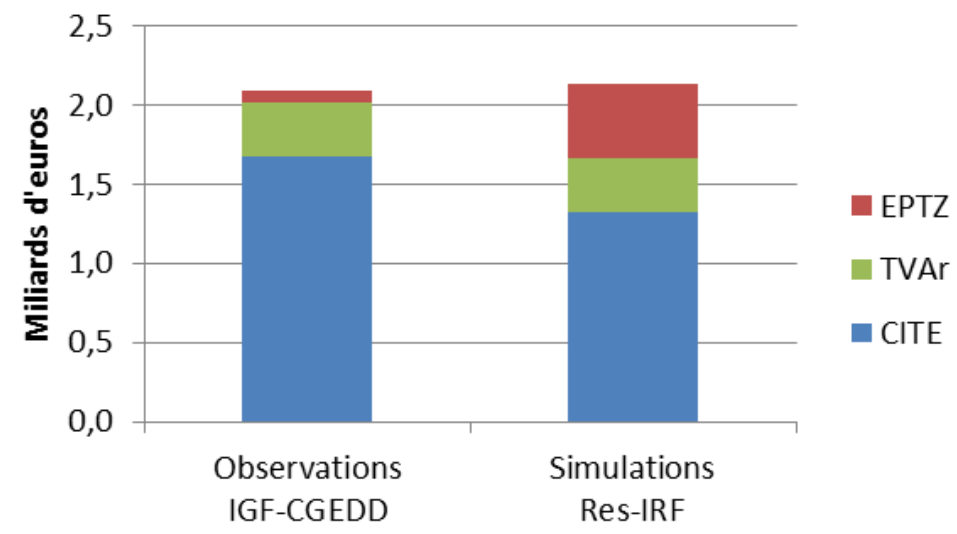

Figure 10: Coût des politiques en 2016

${ }^{11}$ La réglementation thermique est exclue de cette analyse car elle n'entraîne pas de coût public direct. 


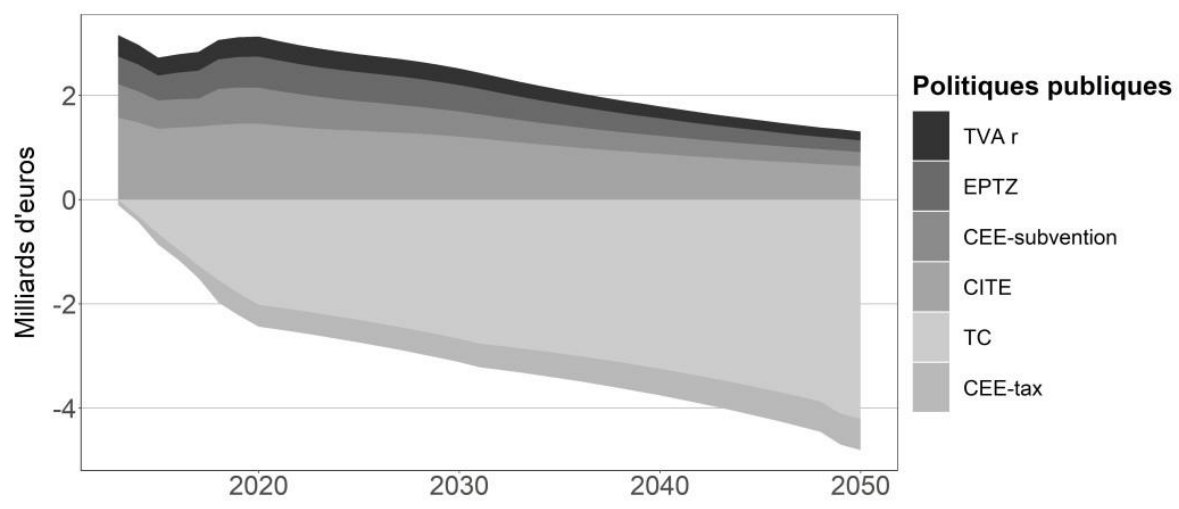

Figure 11: Evolution du coût des politiques

La Figure 12 compare le rapport coût-efficacité de chaque instrument, mesuré selon la méthode décrite à la section 3.3.2. Exprimé en énergie réelle, l'indicateur suggère que la taxe sur le carbone est moins efficiente que les subventions. Les subventions permettent d'économiser l'énergie à un coût de 0,05 à 0,08€ par kWh cumac en 2015 ; le CITE est la moins efficace d'entre elles. L'indicateur augmente au cours du temps à mesure que le potentiel d'économies d'énergie se réduit. L'indicateur offre une image différente lorsqu'il est exprimé en énergie réelle, avec notamment une inversion de l'ordre de mérite entre taxe et subventions. Comme discuté à la section 3.3.2, cette inversion est due au fait que la taxe induit des variations de l'intensité d'utilisation non prises en compte par l'indicateur en énergie conventionnelle. Dans le même ordre d'idées, on constate que, contrairement à d'autres subventions le rapport coût-efficacité des CEE diminue au cours du temps lorsque celui-ci est mesuré en énergie réelle. Ce résultat s'explique par la composante taxe de l'instrument, qui produit des économies d'énergie supplémentaires qui ne sont pas attribuables à la subvention dont le coût est considéré dans la construction de l'indice. ${ }^{12}$

${ }^{12}$ Si le dispositif était limité au secteur considéré ici, le coût des subventions serait égal aux recettes des taxes. 


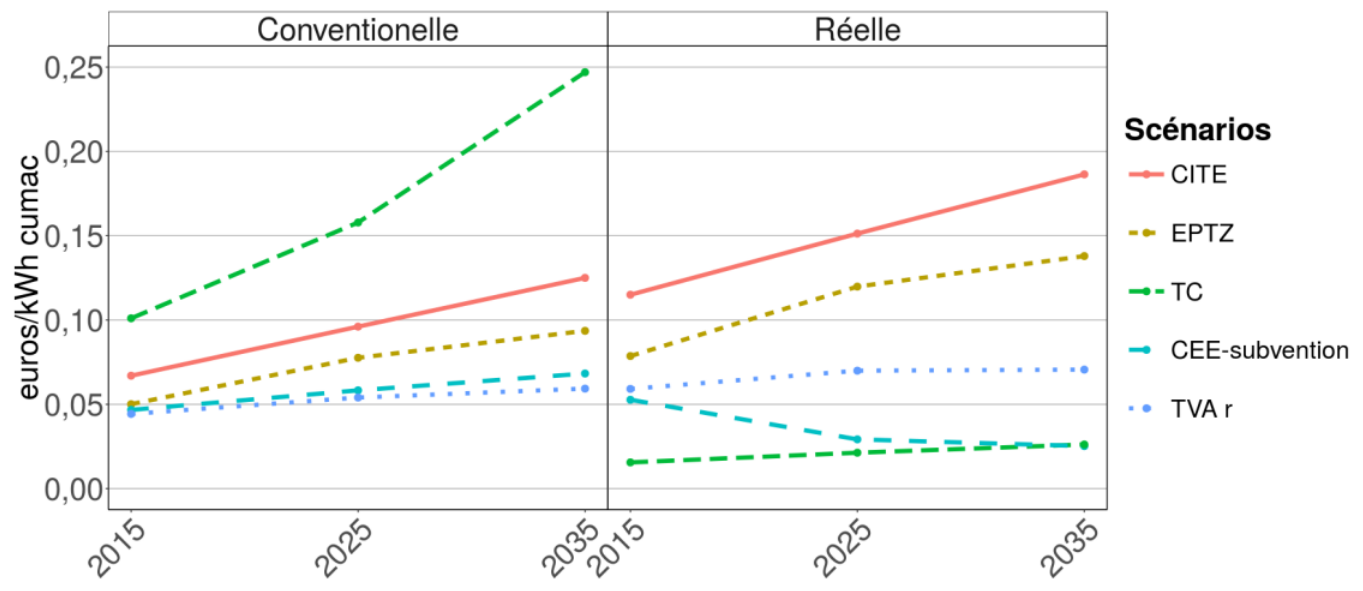

Figure 12: Coût-efficacité des politiques

La Figure 13 confirme ces résultats à l'aide d'un autre indicateur d'efficience : l'effet levier. Rappelons qu'un effet levier élevé et un coût par kWh économisé faible sont tous deux synonyme d'une efficience élevée. Toutefois, l'effet levier diminue moins vite que n'augmente le coût par kWh économisé, ce qui peut s'expliquer par la spécification marginalement croissante des courbes de coût d'efficacité énergétique intégrées au modèle. L'effet levier des subventions varie de 1 à 1,4 en 2015, ce qui est conforme aux estimations disponibles pour d'autres politiques publiques (par exemple, Gobillon et al., 2005 ; Lentile et Mairesse, 2009). Elle est également cohérente avec la similitude d'ordre de grandeur entre les indices de coût-efficacité et les coûts moyens incorporés dans le modèle.

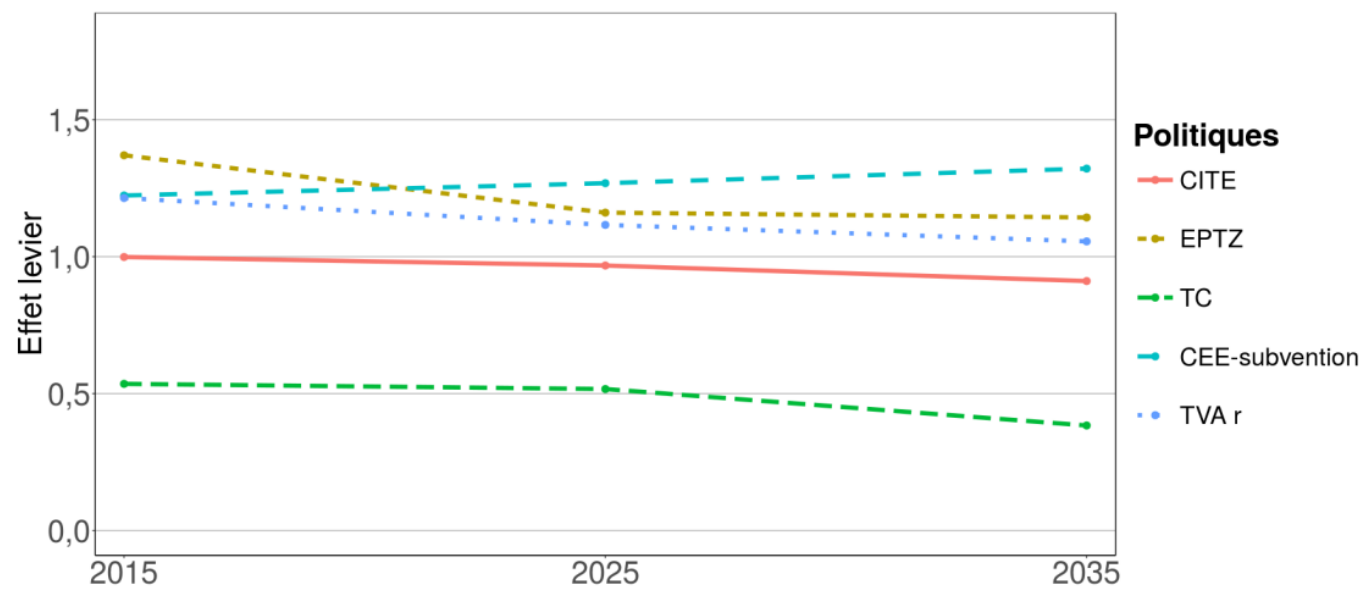

Figure 13: Effet levier des politiques 
Les différents régimes de subvention considérés - taux ad valorem uniforme pour le CITE, ad valorem uniforme et fixé à un niveau plus bas pour la TVA réduite, ad valorem et ciblé pour l'EPTZ ou encore ad valorem régressif pour les CEE - rendent la comparaison entre instruments difficile. Afin de mieux comprendre l'ordre du mérite exposé dans les Figures 12 et 13, et en particulier pour séparer les effets de forme du barème et de niveau, nous nous concentrons sur un instrument, le CITE, dont nous simulons des variantes supplémentaires. Outre les variantes de référence et CITE+ (qui reprend de ciblage performance de l'EPTZ), nous considérons les variantes suivantes: I'une avec un taux ad valorem uniforme mais réduit de moitié (analogue au taux faible de la TVA réduite) ; une autre ciblant les ménages en situation de précarité énergétique, où l'éligibilité est restreinte aux deux premiers quintiles de revenu des ménages; et une dernière qui combine les ciblages performance et précarité. L'effet levier qui en résulte, illustré à la Figure 14, suggèrent que ces différents ajustements ont tous pour effet d'accroître l'efficience. Ces ajustements opèrent cependant par des canaux différents :

- L'effet levier légèrement plus élevé induit par une baisse du taux uniforme est dû à une réorientation des investissements vers les mesures les moins coûteuses. Cet effet contribue à expliquer la différence de performance entre la TVA réduite et le CITE qui s'observe à la Figure 13.

- L'effet levier plus important induit par le ciblage sur la précarité est également dû à une réorientation de l'investissement vers les mesures les moins coûteuses, en vertu de la corrélation entre le revenu et l'efficacité énergétique illustrée à la Figure 1. Elle ouvre la voie à des politiques conciliant efficacité économique et réduction de la précarité énergétique.

- L'augmentation de l'effet levier induite par le ciblage performance est plus persistante que les deux premiers ajustements. Elle s'explique par un fort effet sur la marge intensive d'investissement. Cet effet du ciblage performance, conjugué à celui d'un taux uniforme plus faible, contribue à expliquer la différence de performance entre le CITE et l'EPTZ qui s'observe à la Figure 13.

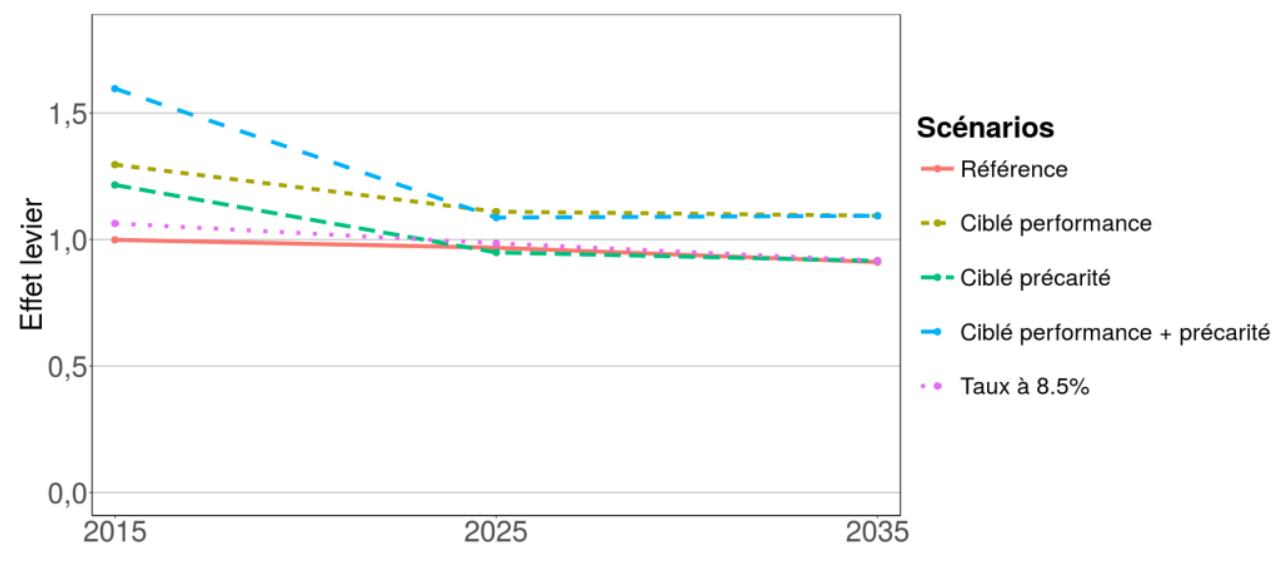

Figure 14: Effet levier avec d'autres variantes du CITE 


\section{Conclusion}

Cette étude quantifie l'impact à moyen et long terme des politiques de rénovation énergétique des logements en France. Elle utilise la dernière version de Res-IRF, un modèle de demande d'énergie offrant une description riche du parc de logements et des comportements des ménages. Le modèle couvre la demande d'électricité, de gaz naturel, de fioul domestique et de bois-énergie pour le chauffage des résidences principales, soit $18 \%$ de la consommation finale d'énergie en France. La pertinence du modèle pour l'étude envisagée est confortée par sa capacité à reproduire les ordres de grandeur et tendances de long terme observées dans le secteur du logement.

L'analyse se concentre sur l'impact en équilibre partiel des différentes aides à l'efficacité énergétique crédit d'impôt pour la transition énergétique, éco-prêt à taux zéro, TVA réduite et certificats d'économies d'énergie -, de la taxe carbone et de la future réglementation thermique de 2020. Le modèle reproduit fidèlement les statistiques disponibles sur le coût des politiques, à l'exception de I'EPTZ, dont l'impact est surestimé d'un ordre de grandeur. Cette surestimation compense dans une certaine mesure la non-prise en compte d'autres politiques, comme les subventions accordées par l'Agence nationale de l'habitat (ANAH) aux ménages à faibles revenus et les récentes campagnes d'information. On peut donc considérer que l'étude fournit une évaluation crédible des trajectoires d'amélioration énergétique vis-à-vis des différents objectifs fixés par l'Etat dans le secteur résidentiel.

De manière générale, l'atteinte des objectifs fixés nécessite de fixer les différents instruments à leur niveau le plus ambitieux et les maintenir jusqu'en 2050. En particulier, les critères d'éligibilité des subventions doivent être redéfinis pour mieux cibler le parc locatif privé, qui demeure à l'écart de la dynamique générale de rénovation. L'évaluation produit des résultats mitigés quant à l'objectif des 500 000 rénovations annuelles : si cet objectif est nettement dépassé au niveau agrégé, la fraction qui doit s'appliquer au logement social est loin d'être atteinte. Cette situation souligne la nécessité pour les autorités de définir une métrique claire pour la comptabilisation des rénovations énergétique afin de faciliter l'évaluation de cet objectif politiquement sensible ${ }^{13}$.

La taxe carbone s'avère être la politique la plus efficace en termes d'économies d'énergie, mais aussi la plus régressive au sens où elle accroît la précarité énergétique (du moins en l'absence d'hypothèse particulière sur l'affectation de ses recettes). Les interactions politiques induisent une variation de l'ordre de 10 \% de l'efficacité d'un instrument. Le CITE apparaît comme la subvention la plus efficace, mais aussi la moins efficiente. L'effet levier des subventions est conforme à celui d'autres subventions publiques documentées dans la littérature. II peut être amélioré en augmentant les taux de subvention de façon inversement proportionnelle au revenu, les ménages modestes étant sur-représentés dans les catégories de logement les moins efficaces. Un tel ciblage permet de concilier efficience économique et justice sociale. En considérant l'ensemble des politiques, le solde budgétaire des recettes de taxe et coût des subventions devrait être excédentaire à partir de 2025. Cette perspective ouvre la voie à une meilleure coordination des dépenses et recettes fiscales liées à la rénovation énergétique. Dans une extension en cours, on considère différents modes de recyclage de la taxe carbone - sous forme de

\footnotetext{
${ }^{13}$ Lorsque Nicolas Hulot a démissionné de son poste de ministre du l'écologie le 4 septembre 2018, il a notamment motivé sa décision par le manquement à cet objectif.
} 
chèque vert ou de subvention à l'efficacité énergétique, modélisée comme une subvention unique qui agrègerait tous les dispositifs existants (CITE, TVAr, EPTZ, CEE) en utilisant le régime proportionnel des CEE (Bourgeois et al., 2019). Le recyclage en subvention génère des effets distributifs comparables à ceux du chèque vert, avec l'intérêt supplémentaire de stimuler davantage la rénovation, améliorant ainsi le confort des ménages modestes.

Outre cette extension, l'exercice présenté ici pourrait être prolongé dans au moins trois directions. D'abord, dans la mesure où tous les objectifs ne sont pas atteints, même avec le bouquet de politiques le plus ambitieux, des politiques supplémentaires pourraient s'avérer nécessaires. Des instruments régulièrement évoqués dans le débat public, comme une obligation de rénovation à chaque mutation d'un logement ou une modulation de la fiscalité foncière en fonction de critères de performance énergétique pourraient être modélisés. Ces travaux pourraient s'appuyer sur des simulations analogues réalisées avec la version précédente du modèle et évaluées par rapport aux objectifs désormais obsolètes du Grenelle de l'environnement (Giraudet et al., 2011 ; Fuk Chun Wing et Kiefer, 2015). Ensuite, les barrières cognitives et stratégiques qui entravent le recours à l'EPTZ doivent être prises en compte. Cette tâche nécessite un travail préalable de microéconomie appliquée visant à identifier ces barrières. Enfin, une évaluation plus complète des politiques intègrerait des effets d'équilibre général et une quantification des réductions des émissions de $\mathrm{CO}_{2}$. Là encore, un tel exercice pourrait s'appuyer sur des travaux précédents liant le module Res-IRF au modèle d'équilibre général IMACLIM-R France et un module technico-économique de génération d'électricité en France (Mathy et al., 2015). 


\section{References}

Allaire, D., G. Gaudière, Y. Majchrzak, C. Masi, 2008, « Problématique qualitative et quantitative de la sortie du parc national de bâtiments », Mémoire du Groupe d'Analyse d'Action Publique, ENPC.

Allcott, H., Greenstone, M., 2012. Is There an Energy Efficiency Gap? Journal of Economic Perspectives 26, 3-28. DOI : $10.1257 /$ jep.26.1.3

Ademe, 2015, Chiffres clé Climat, air, énergie. https://www.ademe.fr/sites/default/files/assets/documents/chiffres-clesclimat-air-energie-8705-bd.pdf

Ademe, 2016. $\quad$ Enquête OPEN. Campagne 2015. Travaux réalisés en 2014. http://www.ademe.fr/sites/default/files/assets/documents/open_2015 8679.pdf

Ademe, 2018. Enquête TREMI. Travaux de rénovation énergétique des maisons individuelles. Campagne 2017.

Anderson, S.T., Kellogg, R., Sallee, J.M., 2013. What do consumers believe about future gasoline prices? Journal of Environmental Economics and Management 66, 383-403. DOI: 10.1016/j.jeem.2013.07.002

Aydin, E., Kok, N., Brounen, D., 2017. Energy efficiency and household behavior: the rebound effect in the residential sector. The RAND Journal of Economics 48, 749-782. DOI: 10.1111/1756-2171.12190

Bourgeois, C., Giraudet, L.-G., Quirion, P., 2019. Social-environmental-economic trade-offs associated with carbon-tax revenue recycling, in: Proceedings of the ECEEE 2019 Summer Study. pp. 1365-1372. https://doi.org/https://www.eceee.org/library/conference_proceedings/eceee_Summer_Studies/2019/7-makebuildings-policies-great-again/social-environmental-economic-trade-offs-associated-with-carbon-tax-revenuerecycling/

Branger, F., L.-G. Giraudet, C. Guivarch, P. Quirion, 2015, Global sensitivity analysis of an energy-economy model of the residential building sector, Environmental Modelling \& Software, 70:45-54. DOI: 10.1016/j.envsoft.2015.03.021

Cayla, J.-M., D. Osso, 2013. Does energy efficiency reduce inequalities ? Impact of policies in residential sector on household budget." Proceedings of the ECEEE Summer Study. https://www.eceee.org/library/conference_proceedings/eceee_Summer_Studies/2013/5a-cutting-the-energy-use-ofbuildings-projects-and-technologies/does-energy-efficiency-reduce-inequalities-impact-of-policies-in-residentialsector-on-household-budget/

CGDD, 2015. Un habitat plus compact et moins énergivore : pour quels coûts de construction ? Etudes \& Documents 135. http://temis.documentation.developpement-durable.gouv.fr/docs/Temis/0083/Temis-0083375/22297.pdf

Charlier, D., Legendre, B., 2019. A Multidimensional Approach to Measuring Fuel Poverty. Energy Journal 40, $27-53$. https://doi.org/10.5547/01956574.40.2.bleg

Charlier, D., Risch, A., 2012. Evaluation of the impact of environmental public policy measures on energy consumption and greenhouse gas emissions in the French residential sector. Energy Policy 46, 170-184. https://doi.org/10.1016/j.enpol.2012.03.048

Charlier, D., Risch, A., Salmon, C., 2018. Energy Burden Alleviation and Greenhouse Gas Emissions Reduction: Can We Reach Two Objectives With One Policy? Ecological Economics 143, 294-313. https://doi.org/10.1016/j.ecolecon.2017.07.002

Combet, E., Ghersi, F., Hourcade, J. C., \& Thubin, C. (2010). La fiscalité carbone au risque des enjeux d'équité. Revue française d'économie, 25(2), 59-91.

Fowlie, M., Greenstone, M., Wolfram, C., 2018. Do Energy Efficiency Investments Deliver? Evidence from the Weatherization Assistance Program. Q J Econ 133, 1597-1644. https://doi.org/10.1093/qje/qjy005

Gerarden, T.D., Newell, R.G., Stavins, R.N., 2017. Assessing the Energy-Efficiency Gap. Journal of Economic Literature 55, 1486-1525. https://doi.org/10.1257/jel.20161360

Giraudet, L.-G., Finon, D., 2015. European experiences with white certifirecate obligations: A critical review of existing evaluations. Economics of Energy \& Environmental Policy 4. DOI : 10.5547/2160-5890.4.1.lgir

Gillingham, K., Newell, R.G., Palmer, K., 2009. Energy Efficiency Economics and Policy. Annual Review of Resource Economics 1, 597-620. DOI: 10.1146/annurev.resource.102308.124234

Giraudet, L.-G., C. Guivarch, P. Quirion, 2011, "Comparing and combining energy saving policies : will proposed residential sector policies meet French official targets ?", The Energy Journal, 32(SI1):213-242. DOI: 10.5547/ISSN0195-6574-EJ-Vol32-SI1-12

Giraudet, L.-G., C. Guivarch, P. Quirion, 2012. Exploring the potential for energy conservation in French households through hybrid modelling, Energy Economics, 34(2):426-445. DOI: 10.1016/j.eneco.2011.07.010

Giraudet, L.-G., Quirion, P., 2008. Efficiency and distributional impacts of tradable white certificates compared to taxes, subsidies and regulations. Revue d'économie politique Vol. 118, 885-914. 
Giraudet, L.-G., Houde, S., Maher, J., 2018. Moral Hazard and the Energy Efficiency Gap: Theory and Evidence. Journal of the Association of Environmental and Resource Economists 5, 755-790. https://doi.org/10.1086/698446

Glotin, D., C. Bourgeois, L.-G. Giraudet, P. Quirion, 2019. Prediction is difficult, even when it's about the past: a hindcast experiment using Res-IRF, an integrated energy-economy model. Energy Economics, DOI: 10.1016/j.eneco.2019.07.012

Gobillon, L., Blanc, D.L., 2005. Quelques effets économiques du prêt à taux zéro. Economie et statistique 381, 63-89. DOI : 10.3406/estat.2005.7209

Hirst, E., Goeltz, R., 1985. Estimating energy savings due to conservation programmes: The BPA residential weatherization pilot programme. Energy Economics 7, 20-28. https://doi.org/10.1016/0140-9883(85)90035-0

IGF et CGEDD, 2017. Revue des dépenses - Aides à la rénovation énergétique des logements privés. http://www.igf.finances.gouv.fr/files/live/sites/igf/files/contributed/IGF\%20internet/2.RapportsPublics/2017/2016M-114.pdf

Insee, 2006. Projections de population pour la France métropolitaine à l'horizon 2050 : la population continue de croître et le vieillissement se poursuit. Insee Première ${ }^{\circ}$ 1089. https://www.insee.fr/fr/statistiques/1280826

Jaffe, A.B., Stavins, R.N., 1994. The energy-efficiency gap: What does it mean? Energy Policy 22, 804-810. DOI: $10.1016 / 0301-4215(94) 90138-4$

Labandeira, X., Labeaga, J.M., López-Otero, X., 2017. A meta-analysis on the price elasticity of energy demand. Energy Policy 102, 549-568. 10.1016/j.enpol.2017.01.002

Lentile, D., J. Mairesse, J., 2009. A policy to boost R\&D: Does the R\&D tax credit work? EIB Papers, 14(1):144-169. https://www.econstor.eu/handle/10419/44906Leray, T., B. de la Roncière, 2002, 30 ans de maîtrise de l'énergie, Association technique énergie environnement, Arcueil

Martin, Y., Y. Carsalade, J.-P. Leteurtrois, F. Moisan, 1998, La maîtrise de l'énergie : rapport d'évaluation, Paris: La Documentation française

Mathy, S., Fink, M., Bibas, R., 2015. Rethinking the role of scenarios: Participatory scripting of low-carbon scenarios for France. Energy Policy 77, 176-190. DOI : 10.1016/j.enpol.2014.11.002

McCollum, D.L., Wilson, C., Pettifor, H., Ramea, K., Krey, V., Riahi, K., Bertram, C., Lin, Z., Edelenbosch, O.Y., Fujisawa, S., 2017. Improving the behavioral realism of global integrated assessment models: An application to consumers' vehicle choices. Transportation Research Part D: Transport and Environment 55, 322-342. https://doi.org/10.1016/j.trd.2016.04.003

Metcalf, G.E., Hassett, K.A., 1999. Measuring the Energy Savings from Home Improvement Investments: Evidence from Monthly Billing Data. Review of Economics and Statistics 81, 516-528. https://doi.org/10.1162/003465399558274

Mundaca, L., Neij, L., Worrell, E., McNeil, M., 2010. Evaluating Energy Efficiency Policies with Energy-Economy Models. Annual Review of Environment and Resources 35, 305-344. https://doi.org/10.1146/annurev-environ$\underline{052810-164840}$

Nauleau, M.-L., 2014. Free-riding on tax credits for home insulation in France: An econometric assessment using panel data. Energy Economics 46, 78-92. https://doi.org/10.1016/j.eneco.2014.08.011

ONPE, 2016. Les chiffres-clés de la précarité énergétique. Edition $\mathrm{n}^{\circ} 2$. http://www.onpe.org/sites/default/files/pdf/tableau_de_bord/chiffres-cles-precarite-energetique-novembre2016.pdf

Sorrell, S., 2004. Understanding barriers to energy efficiency, in: Sorrell, S., O’Malley, E., Schleich, J., Scott, S. (Eds.), The Economics of Energy Efficiency: Barriers to Cost-Effective Investment. Edward Elgar, Cheltenham, pp. 25-93.

Sorrell, S., Dimitropoulos, J., Sommerville, M., 2009. Empirical estimates of the direct rebound effect: A review. Energy Policy 37, 1356-1371. https://doi.org/10.1016/j.enpol.2008.11.026

Sunikka-Blank, M., Galvin, R., 2012. Introducing the prebound effect: the gap between performance and actual energy consumption. Building Research \& Information 40, 260-273. 10.1080/09613218.2012.690952 